\title{
Ginger on Human Health: A Comprehensive Systematic Review of 109 Randomized Controlled Trials
}

\author{
Nguyen Hoang Anh ${ }^{1}$, Sun Jo Kim ${ }^{1}$, Nguyen Phuoc Long ${ }^{1}{ }^{\circledR}$, Jung Eun Min ${ }^{1}$, \\ Young Cheol Yoon ${ }^{1}$, Eun Goo Lee ${ }^{1}$, Mina Kim ${ }^{1}$, Tae Joon Kim ${ }^{1}$, Yoon Young Yang ${ }^{1}$, \\ Eui Young Son ${ }^{1}$, Sang Jun Yoon ${ }^{1}$, Nguyen Co Diem ${ }^{2}$, Hyung Min Kim ${ }^{1}$ and \\ Sung Won Kwon 1 ,*iD \\ 1 College of Pharmacy, Seoul National University, Seoul 08826, Korea; 2018-23140@snu.ac.kr (N.H.A.); \\ danielkim27@snu.ac.kr (S.J.K.); phuoclong@snu.ac.kr (N.P.L.); mje0107@snu.ac.kr (J.E.M.); \\ yunyochl@snu.ac.kr (Y.C.Y.); piggypet@snu.ac.kr (E.G.L.); alsdk@snu.ac.kr (M.K.); joont@snu.ac.kr (T.J.K.); \\ yyyang95@snu.ac.kr (Y.Y.Y.); dmldud3110@snu.ac.kr (E.Y.S.); supercanboy@snu.ac.kr (S.J.Y.); \\ snuhmkim04@snu.ac.kr (H.M.K.) \\ 2 School of Medicine, Vietnam National University, Ho Chi Minh City 70000, Vietnam; \\ ncdiem.stu15@medvnu.edu.vn \\ * Correspondence: swkwon@snu.ac.kr; Tel.: +82-2-880-7844
}

Received: 4 December 2019; Accepted: 31 December 2019; Published: 6 January 2020

\begin{abstract}
Clinical applications of ginger with an expectation of clinical benefits are receiving significant attention. This systematic review aims to provide a comprehensive discussion in terms of the clinical effects of ginger in all reported areas. Following the preferred reporting items for systematic reviews and meta-analyses (PRISMA) guideline, randomized controlled trials on the effects of ginger were investigated. Accordingly, 109 eligible papers were fully extracted in terms of study design, population characteristics, evaluation systems, adverse effects, and main outcomes. The reporting quality of the included studies was assessed based on the Cochrane Collaboration's tool for assessing the risk of bias in randomized trials and integrated together with studies that investigated the same subjects. The included studies that examined the improvement of nausea and vomiting in pregnancy, inflammation, metabolic syndromes, digestive function, and colorectal cancer's markers were consistently supported, whereas other expected functions were relatively controversial. Nevertheless, only 43 clinical trials (39.4\%) met the criterion of having a 'high quality of evidence.' In addition to the quality assessment result, small populations and unstandardized evaluation systems were the observed shortcomings in ginger clinical trials. Further studies with adequate designs are warranted to validate the reported clinical functions of ginger.
\end{abstract}

Keywords: ginger; human health; randomized controlled trials; systematic review

\section{Introduction}

Ginger (Zingiber officinale Roscoe), a well-known herbaceous plant, has been widely used as a flavoring agent and herbal medicine for centuries. Furthermore, the consumption of the ginger rhizome is a typical traditional remedy to relieve common health problems, including pain, nausea, and vomiting [1]. Notably, a prominent number of randomized clinical trials (RCTs) have been conducted to examine ginger's antiemetic effect in various conditions such as motion sickness, pregnancy, and post-anesthesia [2-4]. More than approximately 100 compounds have reportedly been isolated from ginger [5]. Specifically, the major classes of ginger compounds are gingerol, shogaols, zingiberene, and zingerone, as well as other less common compounds, including terpenes, vitamins, 
and minerals [6]. Among them, gingerols are considered as the primary components, reported to possess several bioactivities [7]. As a result, many related biological activities have been explored such as those of antioxidant, antimicrobial, and anti-neuroinflammation, just to name a few [8]. Moreover, in recent years, the role of ginger has been extended to anticancer, chemotherapy-induced nausea and vomiting (CINV), and fatigue, as well as improvements in the quality of life in daily human work $[9,10]$.

These potential pharmacological and physiological activities have led to a significant increase in the number of investigations on the health benefits of ginger. Regarding clinical aspects, there has been a trend of accumulative evidence in terms of ginger efficacy on human health. Indeed, a remarkable number of RCTs that have aimed to discover the benefits of ginger by reducing symptoms have been conducted. For example, multiple RCTs evaluated the effectiveness of ginger supplementation in reducing CINV in cancer patients, as well as in dysmenorrhea [11]. Moreover, several systematic reviews and meta-analysis (SR-MA), which aimed to assess the clinical ginger effectiveness, have been completed. In particular, Chen et al. conducted an SR-MA of oral ginger intake and found that ginger could effectively control menstrual pain in dysmenorrhea [11]. Another SR-MA study revealed that ginger improved lipid profiles and benefited the glucose control, insulin sensitivity, and glycosylated hemoglobin of type 2 diabetes mellitus [12]. In addition, ginger's potency has been regularly proposed in arthritis, gastric dysfunction, and cancers $[6,13,14]$.

Though several systematic reviews have been conducted, limitations regarding the reporting quality still persist. Important subjects that need further investigation include, but are not limited to, heterogeneous population, less stringent criteria, inadequate quality assessment, and inconsistent results. More importantly, there is still a lack of a comprehensive review in terms of critically assessing and comparing the quality of the evidence derived from RCTs in different domains of their efficacy. This study aimed to provide a systematic summarization of the effectiveness of oral ginger in human health and diseases in current RCTs. In addition, we evaluated ginger efficacy in every reported clinical-related aspect to provide future directions for the clinical research of ginger. Furthermore, we evaluated the ongoing development and achievement of ginger-related randomized clinical trials in specific representative topics. Finally, the shortcomings of available RCTs in terms of the ginger effect investigation were discussed.

\section{Materials and Methods}

\subsection{Literature Search Strategy}

The study follows the preferred reporting items for systematic reviews and meta-analyses (PRISMA) guidelines (Table S1) [15]. A systematic search was conducted on six libraries, including four English databases and two Korean databases. Firstly, for English literature, we searched Pubmed, EMBASE, Cochrane Central Register of Controlled Trials (CENTRAL), and Clinical Trials (ClinicalTrial.gov) with the query: "Zingiber officinale" OR "Z. officinale" OR "Ginger." Next, two Korean databases, Korean studies Information Service System (KISS) and National Digital Science Library (NDSL), were searched using the query: "Zingiber officinale" OR "Z. officinale" OR "Ginger" and Korean language terms related to ginger. Data were collected up to July 2019 and regularly updated by manual search. There was no limitation in the search period.

\subsection{Inclusion and Exclusion Criteria}

We first imported the search results to Endnote $X 9$ and performed the duplication removal step. The remaining papers were screened for title and abstract. This study focused on randomized clinical trials investigating the efficacy of ginger to improve human health as well as to support human disease. For that reason, any paper that reported the effectiveness of ginger in clinical aspects was included in this study. Inappropriate articles were excluded for the following reasons: (1) not clinical trials; (2) not related topic; (3) irrelevant data for analysis; (4) secondary analysis; (5) unavailable abstract or full-text; (6) duplication; and (7) case reports, letters, commentaries, meeting records, or review articles. 
At least two authors performed this step to evaluate the eligibility of each item. Next, the qualification of each paper was assessed by reading the full-text, and the qualified articles were collected for the next extraction data step. In addition, a manual search was also conducted by screening the reference lists of the selected articles. Finally, the qualified papers were included for the data extraction process.

\subsection{Data Extraction}

The details of demographic populations and study design information, including year, sample allocation, sample size, age, study design, symptom and disease, treatment and control group intervention information, duration of therapy, and blinding, were extracted. Importantly, we extracted the evaluation outcome system, key findings, and adverse effects of each study, as well as their found side effects, if any.

\subsection{Quality Assessment}

The Cochrane Collaboration's tool was employed to evaluate the risk of bias in individual research for quality assessment [16]. The tool included seven items that aimed to evaluate the quality of study design (e.g., randomization), the result (e.g., outcome reporting), and other biases. All items were independently assessed and scored by at least two reviewers to avoid personal bias. Seven items were evaluated for all 109 studies, which were scored into three scales: $\mathrm{H}$ indicates a high risk of bias, $\mathrm{U}$ indicates an unclear risk of bias, and L indicates a low risk of bias. Additionally, each essential function was scored based on seven quality reporting items: 0 points for a high risk, 1 point for an unclear risk, and 2 points for a low risk of bias, and the quality assessment score (QA score) as the sum of each point. We set the criteria at the QA score of 10 or above as a 'high quality of evidence.'

\section{Results}

\subsection{Study Selection}

After a systematical search that selectively focused on the study design of the clinical trials, we retrieved 221, 222, 59, and five papers from PubMed, Embase, Clinical Trials, and CENTRAL, respectively. Subsequently, 101 duplication records were eliminated. Next, the titles and abstracts of the remaining records were screened for eligibility for the extraction process. Finally, 137 records were included for the full-text monitoring, and 109 qualified articles, including manual search papers remained for the final data extraction step. Similarly, we extended our search method to two Korean literatures databases (KISS and NDSL) and retrieved a remarkable number of studies ( $\mathrm{n}=790$ after duplication removal). Unfortunately, no Korean papers qualified after the standard flow of evaluation. The workflow of this study is shown in Figure 1. 


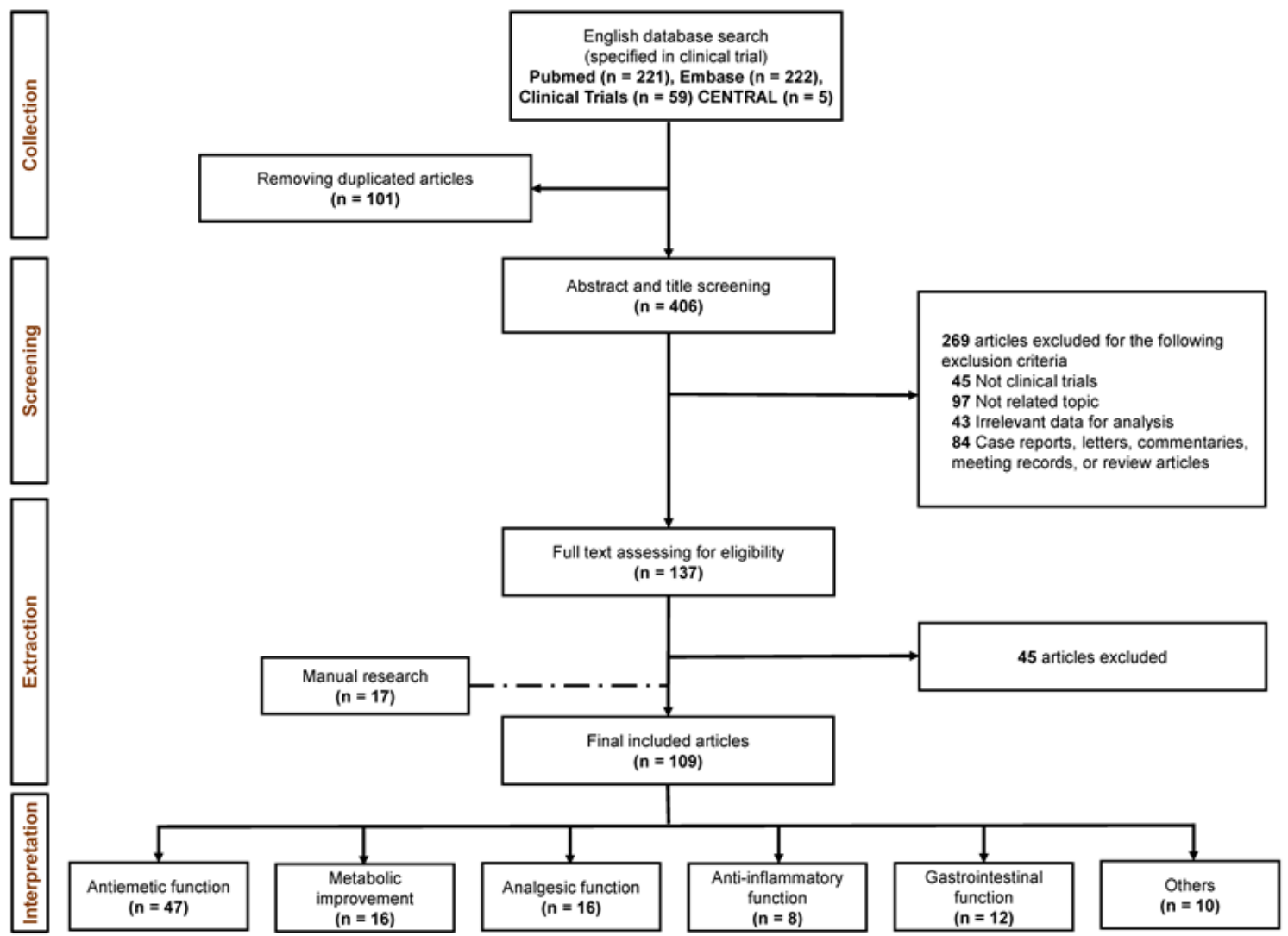

Figure 1. The workflow of systematical search on ginger randomized clinical trials (RCTs) with five categorized substantial functions.

\subsection{Characteristics of Included Studies}

Demographics and RCT designs from the studies that were evaluated to have a 'high quality of evidence' are described in Table 1. The rest of the publications are described in Table S2 due to size of the sample. The trend in the publication of the included studies showed an apparent increase throughout the decades (Figure 2a). Eighteen studies were crossover trials that had a commonly known advantage in reducing the impact of confounding covariates (Figure 2b). Regarding sample size, a population of less than 60 participants per group was mostly conducted (Figure 2c). Seventy-three studies demonstrated the effect of ginger in comparison with placebo groups as a control, while 14 studies compared ginger with medication or other functional materials (Figure 2d). Sixteen studies were designed with both placebo and medication or other functional materials as the control groups (Figure 2d). A daily dosage of 0.5-1.5 g of ginger was frequently adopted, while six studies treated ginger with a multiple dosage range (Figure 2e). The ethnicity of participants in the included studies is summarized in Figure 2f, showing that more than half of the studies were conducted in Iran or the United States. 
Table 1. Study design and demographic description of the included studies with a "high quality of evidence" (quality assessment score is of at least 10).

\begin{tabular}{|c|c|c|c|c|c|c|c|c|c|c|c|}
\hline \multirow{2}{*}{ Author (Year) } & \multirow{2}{*}{$\begin{array}{c}\text { Cohort } \\
\text { Allocation }\end{array}$} & \multirow{2}{*}{ Study Design } & \multirow{2}{*}{$\begin{array}{c}\text { Type of } \\
\text { Disease/Symptom }\end{array}$} & \multicolumn{3}{|c|}{ Intervention } & \multicolumn{3}{|c|}{ Comparator } & \multirow{2}{*}{ Duration } & \multirow{2}{*}{ Blind } \\
\hline & & & & Number & $\mathrm{M} / \mathrm{F}$ & Dosage & Number & $\mathrm{M} / \mathrm{F}$ & Dosage & & \\
\hline $\begin{array}{l}\text { Marx et al. } \\
\text { (2017) [17] }\end{array}$ & Australia & $\begin{array}{l}\text { Randomized } \\
\text { controlled trial }\end{array}$ & $\begin{array}{l}\text { Chemotherapy- } \\
\text { induced nausea } \\
\text { and vomiting }\end{array}$ & 24 & $8 / 16$ & $\begin{array}{l}300 \mathrm{mg} \text { of ginger } \\
\text { extract (5\% } \\
\text { gingerol//cap, } 4 \\
\text { capsules/day }\end{array}$ & 27 & $11 / 16$ & $\begin{array}{c}300 \mathrm{mg} \text { of } \\
\text { placebo/cap, } 4 \\
\text { capsules/day }\end{array}$ & 5 days & Double-blind \\
\hline \multirow[t]{2}{*}{$\begin{array}{l}\text { Sanaati et al. } \\
\text { (2016) [18] }\end{array}$} & Iran & $\begin{array}{l}\text { Randomized } \\
\text { controlled trial }\end{array}$ & $\begin{array}{l}\text { Chemotherapy- } \\
\text { induced nausea } \\
\text { and vomiting }\end{array}$ & 15 & 0/15 & $\begin{array}{l}500 \mathrm{mg} \text { of powdered } \\
\text { ginger/cap, } 2 \\
\text { capsules/day and } \\
\text { DMA regimen * }\end{array}$ & 15 & 0/15 & DMA regimen * & $\begin{array}{l}5 \text { days before and } \\
5 \text { days after } \\
\text { chemotherapy }\end{array}$ & Double-blind \\
\hline & Iran & $\begin{array}{l}\text { Randomized } \\
\text { controlled trial }\end{array}$ & $\begin{array}{l}\text { Chemotherapy- } \\
\text { induced nausea } \\
\text { and vomiting }\end{array}$ & 15 & 0/15 & $\begin{array}{l}500 \mathrm{mg} \text { of powdered } \\
\text { ginger/cap, } 2 \\
\text { capsules/day and } \\
\text { DMA regimen * }\end{array}$ & 15 & $0 / 15$ & $\begin{array}{c}500 \mathrm{mg} \text { of } \\
\text { powdered } \\
\text { chamomile/cap, } 2 \\
\text { capsules/day and } \\
\text { DMA regimen * }\end{array}$ & $\begin{array}{l}5 \text { days before and } \\
5 \text { days after } \\
\text { chemotherapy }\end{array}$ & Double-blind \\
\hline $\begin{array}{l}\text { Thamlikitkul et al. } \\
\text { (2016) [19] }\end{array}$ & Thailand & $\begin{array}{l}\text { Crossover } \\
\text { randomized } \\
\text { controlled trial }\end{array}$ & $\begin{array}{l}\text { Chemotherapy- } \\
\text { induced nausea } \\
\text { and vomiting }\end{array}$ & $\begin{array}{l}\text { Second cycle: } 19 ; \\
\text { Third cycle: } 15\end{array}$ & $\begin{array}{l}\text { Second cycle: } 0 / 19 \\
\text { Third cycle: 0/15 }\end{array}$ & $\begin{array}{l}500 \mathrm{mg} \text { of powdered } \\
\text { ginger/cap, } 2 \\
\text { capsules/day }\end{array}$ & $\begin{array}{l}\text { Second cycle: } 15 ; \\
\text { Third cycle: } 19\end{array}$ & $\begin{array}{l}\text { Second cycle: 0/15; } \\
\text { Third cycle: 0/19 }\end{array}$ & $\begin{array}{c}500 \mathrm{mg} \text { of } \\
\text { placebo/cap, } 2 \\
\text { capsules/day }\end{array}$ & $\begin{array}{l}5 \text { days at each } \\
\text { second and third } \\
\text { cycle of } \\
\text { chemotherapy }\end{array}$ & Double-blind \\
\hline $\begin{array}{l}\text { Li et al. } \\
\text { (2017) [20] }\end{array}$ & China & $\begin{array}{l}\text { Randomized } \\
\text { controlled trial }\end{array}$ & $\begin{array}{l}\text { Chemotherapy- } \\
\text { induced nausea } \\
\text { and vomiting }\end{array}$ & 71 & $53 / 18$ & $\begin{array}{l}250 \mathrm{mg} \text { of powdered } \\
\text { ginger (5\% } \\
\text { gingerols)/cap, } 2 \\
\text { capsules/day, bid }\end{array}$ & 69 & $47 / 22$ & $\begin{array}{l}250 \mathrm{mg} \text { of corn } \\
\text { starch/cap, } 2 \\
\text { capsules/day, bid }\end{array}$ & 5 days & Double-blind \\
\hline $\begin{array}{l}\text { Ansari et al. } \\
\text { (2016) [21] }\end{array}$ & Iran & $\begin{array}{l}\text { Randomized } \\
\text { controlled trial }\end{array}$ & $\begin{array}{l}\text { Chemotherapy-inducec } \\
\text { nausea and } \\
\text { vomiting }\end{array}$ & ed & $0 / 57$ & $\begin{array}{c}250 \mathrm{mg} \text { of powdered } \\
\text { ginger/cap, } 4 \\
\text { capsules/day, bid }\end{array}$ & 62 & $0 / 62$ & $\begin{array}{c}250 \mathrm{mg} \text { of } \\
\text { starch/cap, } 4 \\
\text { capsules/day, bid }\end{array}$ & $\begin{array}{l}3 \text { days for each } 3 \\
\text { cycles }\end{array}$ & Single-blind \\
\hline \multirow[t]{2}{*}{$\begin{array}{l}\text { Sharifzadeh et al. } \\
\text { (2018) [4] }\end{array}$} & Iran & $\begin{array}{l}\text { Randomized } \\
\text { controlled trial }\end{array}$ & $\begin{array}{c}\text { Nausea and } \\
\text { vomiting because } \\
\text { of pregnancy }\end{array}$ & 28 & $0 / 28$ & $\begin{array}{c}500 \text { mg of ginger/cap } \\
2 \text { capsules/day }\end{array}$ & 26 & $0 / 26$ & $\begin{array}{l}40 \mathrm{mg} \text { of vitamin } \\
\text { B6/cap, } 2 \\
\text { capsules/day }\end{array}$ & 4 days & Triple- blind \\
\hline & Iran & $\begin{array}{l}\text { Randomized } \\
\text { controlled trial }\end{array}$ & $\begin{array}{l}\text { Nausea and } \\
\text { vomiting because } \\
\text { of pregnancy }\end{array}$ & 28 & $0 / 28$ & $\begin{array}{l}500 \mathrm{mg} \text { of ginger/cap } \\
2 \text { capsules/day }\end{array}$ & 23 & $0 / 23$ & $\begin{array}{c}2 \text { placebo } \\
\text { capsules/day }\end{array}$ & 4 days & Triple-blind \\
\hline $\begin{array}{l}\text { Matsumura et al. } \\
\text { (2015) [22] }\end{array}$ & $\begin{array}{l}\text { United } \\
\text { States }\end{array}$ & $\begin{array}{l}\text { Randomized } \\
\text { controlled trial }\end{array}$ & $\begin{array}{l}\text { Muscle damage } \\
\text { and delayed onset } \\
\text { muscle soreness }\end{array}$ & 10 & $5 / 5$ & $\begin{array}{c}4 \mathrm{~g} \text { of powdered } \\
\text { ginger in } \\
\text { capsule(s)/day }\end{array}$ & 10 & $5 / 5$ & $\begin{array}{l}4 \mathrm{~g} \text { of dextrose in } \\
\text { capsule(s)/day }\end{array}$ & 5 days & Double-blind \\
\hline $\begin{array}{l}\text { Martins et al. } \\
(2018)[23]\end{array}$ & Brazil & $\begin{array}{l}\text { Randomized } \\
\text { controlled trial }\end{array}$ & Migraine & 30 & $4 / 26$ & $\begin{array}{l}200 \mathrm{mg} \text { of ginger } \\
\text { extract/cap, } 2 \\
\text { capsules/dose }+100 \\
\text { mg of ketoprofen (i.v.) }\end{array}$ & 30 & $4 / 26$ & $\begin{array}{c}200 \mathrm{mg} \text { of } \\
\text { cellulose/cap, } 2 \\
\text { capsules/dose }+ \\
100 \mathrm{mg} \text { of } \\
\text { ketoprofen (i.v.) }\end{array}$ & Single dose & Double-blind \\
\hline $\begin{array}{l}\text { Arzati et al. } \\
\text { (2017) [24] }\end{array}$ & Iran & $\begin{array}{l}\text { Randomized } \\
\text { controlled trial }\end{array}$ & $\begin{array}{l}\text { Type } 2 \text { diabetes } \\
\text { mellitus }\end{array}$ & 25 & 9/16 & $\begin{array}{l}500 \mathrm{mg} \text { of ginger/cap } \\
4 \text { capsules/day }\end{array}$ & 25 & $7 / 18$ & $\begin{array}{l}500 \mathrm{mg} \text { of wheat } \\
\text { flour/cap, } 4 \\
\text { capsules/day }\end{array}$ & 10 weeks & Double-blind \\
\hline $\begin{array}{l}\text { Attari et al. } \\
\text { (2016) [25] }\end{array}$ & Iran & $\begin{array}{l}\text { Randomized } \\
\text { controlled trial }\end{array}$ & Obesity & 39 & $0 / 39$ & $\begin{array}{c}1 \mathrm{~g} \text { of powdered } \\
\text { ginger/tab, } 2 \\
\text { tablets/day }\end{array}$ & 31 & $0 / 31$ & $\begin{array}{l}1 \mathrm{~g} \text { of corn starch } \\
\text { and other } \\
\text { excipients/tab, } \\
\text { 2tablets/day }\end{array}$ & 12 weeks & Double-blind \\
\hline
\end{tabular}


Table 1. Cont.

\begin{tabular}{|c|c|c|c|c|c|c|c|c|c|c|c|}
\hline \multirow{2}{*}{ Author (Year) } & \multirow{2}{*}{$\begin{array}{c}\text { Cohort } \\
\text { Allocation }\end{array}$} & \multirow{2}{*}{ Study Design } & \multirow{2}{*}{$\begin{array}{c}\text { Type of } \\
\text { Disease/Symptom }\end{array}$} & \multicolumn{3}{|c|}{ Intervention } & \multicolumn{3}{|c|}{ Comparator } & \multirow{2}{*}{ Duration } & \multirow{2}{*}{ Blind } \\
\hline & & & & Number & $\mathrm{M} / \mathrm{F}$ & Dosage & Number & $\mathrm{M} / \mathrm{F}$ & Dosage & & \\
\hline $\begin{array}{l}\text { Attari et al. } \\
\text { (2015) [26] }\end{array}$ & Iran & $\begin{array}{l}\text { Randomized } \\
\text { controlled trial }\end{array}$ & $\begin{array}{c}\text { Obesity } \\
\text { management }\end{array}$ & 39 & $0 / 39$ & $\begin{array}{c}1 \mathrm{~g} \text { of powdered } \\
\text { ginger/tab, } 2 \\
\text { tablets/day }\end{array}$ & 31 & $0 / 31$ & $\begin{array}{l}1 \mathrm{~g} \text { of corn } \\
\text { starch/tab, } 2 \\
\text { tablets/day }\end{array}$ & 12 weeks & Double-blind \\
\hline $\begin{array}{c}\text { Mozaffari-Khosravi } \\
\text { et al. } \\
\text { (2016) [13] }\end{array}$ & Iran & $\begin{array}{l}\text { Randomized } \\
\text { controlled trial }\end{array}$ & Knee osteoarthritis & 50 & $3 / 47$ & $\begin{array}{l}500 \mathrm{mg} \text { of powdered } \\
\text { ginger/cap, } 2 \\
\text { capsules/day } \\
\end{array}$ & 50 & $7 / 43$ & $\begin{array}{l}500 \mathrm{mg} \text { of } \\
\text { starch/cap, } 2 \\
\text { capsules/day }\end{array}$ & 3 months & Double-blind \\
\hline $\begin{array}{l}\text { Aryaeian et al. } \\
\text { (2019) [27] }\end{array}$ & Iran & $\begin{array}{l}\text { Randomized } \\
\text { controlled trial }\end{array}$ & $\begin{array}{l}\text { Active rheumatoid } \\
\text { arthritis }\end{array}$ & 33 & $4 / 29$ & $\begin{array}{l}750 \mathrm{mg} \text { of powdered } \\
\text { ginger/cap, } 2 \\
\text { capsules/day }\end{array}$ & 30 & $3 / 27$ & $\begin{array}{l}750 \mathrm{mg} \text { of wheat } \\
\text { flour/cap, } 2 \\
\text { capsules/day }\end{array}$ & 12 weeks & Double-blind \\
\hline $\begin{array}{l}\text { Kashefi et al. } \\
\text { (2015) [28] }\end{array}$ & Iran & $\begin{array}{l}\text { Randomized } \\
\text { controlled trial }\end{array}$ & $\begin{array}{l}\text { Heavy menstrual } \\
\text { bleeding }\end{array}$ & $\begin{array}{l}43 \text { (1st month); } \\
41 \text { (2nd month); } \\
38 \text { (3rd month) }\end{array}$ & $\begin{array}{l}\text { 0/43 (1st month) } \\
0 / 41 \text { (2nd month) } \\
0 / 38 \text { (3rd month) }\end{array}$ & $\begin{array}{l}250 \mathrm{mg} \text { of powdered } \\
\text { ginger/cap, } 3 \\
\text { capsules/day }\end{array}$ & $\begin{array}{l}43 \text { (1st month); } \\
38 \text { (2nd month); } \\
33 \text { (3rd month) }\end{array}$ & $\begin{array}{l}\text { 0/43 (1st month) } \\
0 / 38 \text { (2nd month) } \\
0 / 33 \text { (3rd month) }\end{array}$ & $\begin{array}{l}250 \mathrm{mg} \text { of } \\
\text { lactose/cap, } 3 \\
\text { capsules/day }\end{array}$ & $\begin{array}{l}\text { From the day } \\
\text { before menstrual } \\
\text { bleeding to the } 3 \text { rd } \\
\text { day of the } \\
\text { menstrual period }\end{array}$ & Double-blind \\
\hline $\begin{array}{l}\text { Paritakul et al. } \\
\text { (2016) [29] }\end{array}$ & Thailand & $\begin{array}{l}\text { Randomized } \\
\text { controlled trial }\end{array}$ & $\begin{array}{c}\text { Breast milk } \\
\text { volume of } \\
\text { postpartum } \\
\text { women who } \\
\text { delivered a term } \\
\text { baby }(\geq 37 \text { weeks } \\
\text { of gestation) }\end{array}$ & 15 & 0/15 & $\begin{array}{l}500 \mathrm{mg} \text { of powdered } \\
\text { ginger/cap, } 2 \\
\text { capsules/day }\end{array}$ & 21 & $0 / 21$ & $\begin{array}{l}500 \mathrm{mg} \text { of corn } \\
\text { starch/cap, } 2 \\
\text { capsules/day }\end{array}$ & 7 days & Double-blind \\
\hline \multirow[t]{3}{*}{$\begin{array}{l}\text { Ryan et al. } \\
\text { (2012) [30] }\end{array}$} & $\begin{array}{l}\text { United } \\
\text { States }\end{array}$ & $\begin{array}{l}\text { Randomized } \\
\text { controlled trial }\end{array}$ & $\begin{array}{l}\text { Chemotherapy-induced } \\
\text { nausea and } \\
\text { vomiting }\end{array}$ & 134 & $12 / 122$ & $\begin{array}{l}\text { One ginger capsule } \\
(250 \mathrm{mg} \text { of ginger } \\
\text { extract) + } 2 \text { placebo } \\
\text { capsules, twice/day }\end{array}$ & 149 & $14 / 135$ & $\begin{array}{l}\text { Three placebo } \\
\text { capsules, } \\
\text { twice/day }\end{array}$ & 6 days & Double-blind \\
\hline & $\begin{array}{l}\text { United } \\
\text { States }\end{array}$ & $\begin{array}{l}\text { Randomized } \\
\text { controlled trial }\end{array}$ & $\begin{array}{l}\text { Chemotherapy-induced } \\
\text { nausea and } \\
\text { vomiting }\end{array}$ & 141 & 19/122 & $\begin{array}{l}\text { Two ginger capsules } \\
(250 \mathrm{mg} \text { of ginger } \\
\text { extract) + 1 placebo } \\
\text { capsule, twice/day }\end{array}$ & & & & & \\
\hline & $\begin{array}{l}\text { United } \\
\text { States }\end{array}$ & $\begin{array}{l}\text { Randomized } \\
\text { controlled trial }\end{array}$ & $\begin{array}{l}\text { Chemotherapy-induced } \\
\text { nausea and } \\
\text { vomiting } \\
\end{array}$ & 152 & $10 / 142$ & $\begin{array}{l}\text { Three ginger capsules } \\
\text { ( } 250 \mathrm{mg} \text { of ginger } \\
\text { extract), twice/day }\end{array}$ & & & & & \\
\hline \multirow[t]{2}{*}{$\begin{array}{l}\text { Zick et al. } \\
\text { (2008) [31] }\end{array}$} & $\begin{array}{l}\text { United } \\
\text { States }\end{array}$ & $\begin{array}{l}\text { Randomized } \\
\text { controlled trial }\end{array}$ & $\begin{array}{l}\text { Chemotherapy-induced } \\
\text { nausea and } \\
\text { vomiting }\end{array}$ & 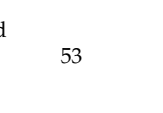 & $14 / 39$ & $\begin{array}{l}250 \mathrm{mg} \text { of dry ginger } \\
\text { root extract/cap, } 4 \\
\text { ginger capsules and } 4 \\
\text { lactose capsules/day }\end{array}$ & 57 & $14 / 43$ & $\begin{array}{c}250 \mathrm{mg} \text { of } \\
\text { lactose/cap, } 8 \\
\text { capsules/day }\end{array}$ & 28 days & Double-blind \\
\hline & $\begin{array}{l}\text { United } \\
\text { States }\end{array}$ & $\begin{array}{l}\text { Randomized } \\
\text { controlled trial }\end{array}$ & $\begin{array}{l}\text { Chemotherapy-induced } \\
\text { nausea and } \\
\text { vomiting }\end{array}$ & 52 & $12 / 40$ & $\begin{array}{l}250 \mathrm{mg} \text { of dry ginger } \\
\text { root extract/cap, } 8 \\
\text { capsules/day }\end{array}$ & & & & & \\
\hline
\end{tabular}


Table 1. Cont.

\begin{tabular}{|c|c|c|c|c|c|c|c|c|c|c|c|}
\hline \multirow{2}{*}{ Author (Year) } & \multirow{2}{*}{$\begin{array}{c}\text { Cohort } \\
\text { Allocation }\end{array}$} & \multirow{2}{*}{ Study Design } & \multirow{2}{*}{$\begin{array}{c}\text { Type of } \\
\text { Disease/Symptom }\end{array}$} & \multicolumn{3}{|c|}{ Intervention } & \multicolumn{3}{|c|}{ Comparator } & \multirow{2}{*}{ Duration } & \multirow{2}{*}{ Blind } \\
\hline & & & & Number & $\mathrm{M} / \mathrm{F}$ & Dosage & Number & $\mathbf{M} / \mathbf{F}$ & Dosage & & \\
\hline $\begin{array}{l}\text { Fahimi et al. } \\
\text { (2010) [32] }\end{array}$ & Iran & $\begin{array}{c}\text { Crossover } \\
\text { randomized } \\
\text { controlled trial }\end{array}$ & $\begin{array}{l}\text { Chemotherapy-induced } \\
\text { nausea and } \\
\text { vomiting }\end{array}$ & 36 & $26 / 10$ & $\begin{array}{l}250 \mathrm{mg} \text { of powdered } \\
\text { ginger/cap, } 2 \\
\text { capsules/day }\end{array}$ & 36 & $26 / 10$ & $\begin{array}{c}250 \mathrm{mg} \text { of } \\
\text { lactose/cap, } 2 \\
\text { capsules/day }\end{array}$ & $\begin{array}{l}3 \text { days for each } \\
\text { period }\end{array}$ & Double-blind \\
\hline $\begin{array}{l}\text { Yekta et al. } \\
\text { (2012) [33] }\end{array}$ & Iran & $\begin{array}{l}\text { Randomized } \\
\text { controlled trial }\end{array}$ & $\begin{array}{l}\text { Chemotherapy-induced } \\
\text { nausea and } \\
\text { vomiting }\end{array}$ & 40 & $0 / 40$ & $\begin{array}{l}250 \mathrm{mg} \text { of powdered } \\
\text { ginger/cap, } 4 \\
\text { capsules/day }\end{array}$ & 40 & $0 / 40$ & $\begin{array}{c}250 \mathrm{mg} \text { of } \\
\text { starch/cap, } 4 \\
\text { capsules/day }\end{array}$ & $\begin{array}{c}6 \text { days from three } \\
\text { days before a } \\
\text { chemotherapy } \\
\text { session }\end{array}$ & Double-blind \\
\hline $\begin{array}{l}\text { Ensiyeh et al. } \\
\text { (2009) [3] }\end{array}$ & Iran & $\begin{array}{l}\text { Randomized } \\
\text { controlled trial }\end{array}$ & $\begin{array}{c}\text { Nausea and } \\
\text { vomiting because } \\
\text { of pregnancy }\end{array}$ & 35 & $0 / 35$ & $\begin{array}{l}500 \mathrm{mg} \text { of powdered } \\
\text { ginger/cap, } 2 \\
\text { capsules/day }\end{array}$ & 34 & $0 / 34$ & $\begin{array}{l}20 \mathrm{mg} \text { of vitamin } \\
\text { B6/cap, } 2 \\
\text { capsules/day }\end{array}$ & 4 days & Double-blind \\
\hline $\begin{array}{l}\text { Willetts et al. } \\
\text { (2003) [34] }\end{array}$ & Australia & $\begin{array}{l}\text { Randomized } \\
\text { controlled trial }\end{array}$ & $\begin{array}{l}\text { Nausea and } \\
\text { vomiting because } \\
\text { of pregnancy }\end{array}$ & 48 & $0 / 48$ & $\begin{array}{l}125 \mathrm{mg} \text { of ginger } \\
\text { extract/cap, } 4 \\
\text { capsules/day }\end{array}$ & 51 & 0/51 & $\begin{array}{l}4 \text { capsules/day, } \\
\text { each capsule } \\
\text { containing soya } \\
\text { bean oil }\end{array}$ & 4 days & Double-blind \\
\hline $\begin{array}{l}\text { Vutyavanich et al. } \\
\text { (2001) [35] }\end{array}$ & Thailand & $\begin{array}{l}\text { Randomized } \\
\text { controlled trial }\end{array}$ & $\begin{array}{c}\text { Nausea and } \\
\text { vomiting because } \\
\text { of pregnancy }\end{array}$ & 32 & $0 / 32$ & $\begin{array}{l}250 \mathrm{mg} \text { of powdered } \\
\text { ginger/cap, } 4 \\
\text { capsules/day }\end{array}$ & 38 & $0 / 38$ & $\begin{array}{l}4 \text { placebo } \\
\text { capsules/day }\end{array}$ & 4 days & Double-blind \\
\hline $\begin{array}{l}\text { Fischer-Rasmussen } \\
\text { et al. } \\
\text { (1990) [36] }\end{array}$ & Denmark & $\begin{array}{c}\text { Crossover } \\
\text { randomized } \\
\text { controlled trial }\end{array}$ & $\begin{array}{c}\text { Nausea and } \\
\text { vomiting because } \\
\text { of pregnancy }\end{array}$ & 27 & $0 / 27$ & $\begin{array}{l}250 \mathrm{mg} \text { of powdered } \\
\text { ginger root/cap, } 4 \\
\text { capsules/day }\end{array}$ & 27 & $0 / 27$ & $\begin{array}{l}250 \mathrm{mg} \text { of } \\
\text { lactose/cap, } 4 \\
\text { capsules/day }\end{array}$ & $\begin{array}{c}\text { Two periods of } 4 \\
\text { days }\end{array}$ & Double-blind \\
\hline $\begin{array}{l}\text { Smith et al. } \\
\text { (2004) [37] }\end{array}$ & Australia & $\begin{array}{l}\text { Randomized } \\
\text { controlled trial }\end{array}$ & $\begin{array}{c}\text { Nausea and } \\
\text { vomiting because } \\
\text { of pregnancy }\end{array}$ & 145 & $0 / 145$ & $\begin{array}{l}350 \mathrm{mg} \text { of ginger/cap, } \\
3 \text { capsules/day, tid }\end{array}$ & 146 & $0 / 146$ & $\begin{array}{l}25 \mathrm{mg} \text { of vitamin } \\
\text { B6/cap, } 3 \\
\text { capsules/day, tid }\end{array}$ & 3 weeks & Double-blind \\
\hline $\begin{array}{l}\text { Biswas et al. } \\
\text { (2011) [38] }\end{array}$ & India & $\begin{array}{l}\text { Randomized } \\
\text { controlled trial }\end{array}$ & $\begin{array}{l}\text { Nausea and } \\
\text { vomiting because } \\
\text { of pregnancy }\end{array}$ & 34 & $0 / 34$ & $\begin{array}{l}150 \mathrm{mg} \text { of dried ginger } \\
\text { extract/tab, 3 } \\
\text { tablets/day }\end{array}$ & 29 & $0 / 29$ & $\begin{array}{c}10 \mathrm{mg} \text { of } \\
\text { doxylamine }+10 \\
\mathrm{mg} \text { of } \\
\text { pyridoxine/tab, } 2 \\
\text { or } 3 \text { tablets/day }\end{array}$ & 3 weeks & Single-blind \\
\hline \multirow[t]{2}{*}{$\begin{array}{l}\text { Firouzbakht et al. } \\
\text { (2014) [39] }\end{array}$} & Iran & $\begin{array}{l}\text { Randomized } \\
\text { controlled trial }\end{array}$ & $\begin{array}{c}\text { Nausea and } \\
\text { vomiting because } \\
\text { of pregnancy }\end{array}$ & 24 & $0 / 24$ & $\begin{array}{l}250 \mathrm{mg} \text { of powdered } \\
\text { ginger/cap, } 4 \\
\text { capsules/day } \\
\end{array}$ & 35 & $0 / 35$ & $\begin{array}{c}40 \mathrm{mg} \text { of vitamin } \\
\text { B6/cap, } 4 \\
\text { capsules/day }\end{array}$ & 4 days & Double-bling \\
\hline & Iran & $\begin{array}{l}\text { Randomized } \\
\text { controlled trial }\end{array}$ & $\begin{array}{c}\text { Nausea and } \\
\text { vomiting because } \\
\text { of pregnancy }\end{array}$ & 24 & $0 / 24$ & $\begin{array}{l}250 \mathrm{mg} \text { of powdered } \\
\text { ginger/cap, } 4 \\
\text { capsules/day }\end{array}$ & 28 & $0 / 28$ & $\begin{array}{c}40 \mathrm{mg} \text { of sugar/cap } \\
4 \text { capsules/day }\end{array}$ & 4 days & Double-blind \\
\hline \multirow[t]{2}{*}{$\begin{array}{l}\text { Arfeen et al. } \\
\text { (1995) [40] }\end{array}$} & Australia & $\begin{array}{l}\text { Randomized } \\
\text { controlled trial }\end{array}$ & $\begin{array}{l}\text { Postoperative } \\
\text { nausea and } \\
\text { vomiting }\end{array}$ & 36 & N/A & $\begin{array}{c}\text { One capsule } \\
\text { containing } 500 \mathrm{mg} \text { of } \\
\text { powdered ginger and } \\
\text { one placebo capsule }\end{array}$ & 36 & N/A & $\begin{array}{l}\text { Two placebo } \\
\text { capsules }\end{array}$ & Single-dose & Double-bling \\
\hline & Australia & $\begin{array}{l}\text { Randomized } \\
\text { controlled trial }\end{array}$ & $\begin{array}{l}\text { Postoperative } \\
\text { nausea and } \\
\text { vomiting }\end{array}$ & 36 & N/A & $\begin{array}{c}\text { Two capsules } \\
\text { containing } 500 \mathrm{mg} \text { of } \\
\text { powdered ginger }\end{array}$ & 36 & $\mathrm{~N} / \mathrm{A}$ & $\begin{array}{l}\text { Two placebo } \\
\text { capsules }\end{array}$ & Single-dose & Double-blind \\
\hline
\end{tabular}


Table 1. Cont.

\begin{tabular}{|c|c|c|c|c|c|c|c|c|c|c|c|}
\hline \multirow{2}{*}{ Author (Year) } & \multirow{2}{*}{$\begin{array}{l}\text { Cohort } \\
\text { Allocation }\end{array}$} & \multirow{2}{*}{ Study Design } & \multirow{2}{*}{$\begin{array}{c}\text { Type of } \\
\text { Disease/Symptom }\end{array}$} & \multicolumn{3}{|c|}{ Intervention } & \multicolumn{3}{|c|}{ Comparator } & \multirow{2}{*}{ Duration } & \multirow{2}{*}{ Blind } \\
\hline & & & & Number & $\mathrm{M} / \mathrm{F}$ & Dosage & Number & $\mathbf{M} / \mathbf{F}$ & Dosage & & \\
\hline \multirow[t]{2}{*}{$\begin{array}{l}\text { Eberhart et al. } \\
(2003)[41]\end{array}$} & Germany & $\begin{array}{l}\text { Randomized } \\
\text { controlled trial }\end{array}$ & $\begin{array}{l}\text { Postoperative } \\
\text { nausea and } \\
\text { vomiting }\end{array}$ & 59 & $0 / 59$ & $\begin{array}{c}100 \mathrm{mg} \text { of ginger } \\
\text { extract/cap, } 1 \text { ginger } \\
\text { capsule }+1 \text { placebo } \\
\text { capsule/dose } \\
\end{array}$ & 59 & $0 / 59$ & $\begin{array}{c}2 \text { placebo } \\
\text { capsules/dose }\end{array}$ & $\begin{array}{l}\text { Triple dose: before } \\
\text { operation and } 3 \mathrm{~h} \\
\text { and } 6 \mathrm{~h} \text { post } \\
\text { operation }\end{array}$ & Double-blind \\
\hline & Germany & $\begin{array}{l}\text { Randomized } \\
\text { controlled trial }\end{array}$ & $\begin{array}{l}\text { Postoperative } \\
\text { nausea and } \\
\text { vomiting }\end{array}$ & 57 & $0 / 57$ & $\begin{array}{l}100 \mathrm{mg} \text { of ginger } \\
\text { extract/cap, } 2 \text { ginger } \\
\text { capsules/dose }\end{array}$ & 59 & $0 / 59$ & $\begin{array}{c}2 \text { placebo } \\
\text { capsules/dose }\end{array}$ & $\begin{array}{l}\text { Triple dose: before } \\
\text { operation and } 3 \mathrm{~h} \\
\text { and } 6 \mathrm{~h} \text { post } \\
\text { operation }\end{array}$ & Double-blind \\
\hline $\begin{array}{l}\text { Mandal et al. } \\
\text { (2014) [42] }\end{array}$ & India & $\begin{array}{l}\text { Randomized } \\
\text { controlled trial }\end{array}$ & $\begin{array}{l}\text { Postoperative } \\
\text { nausea and } \\
\text { vomiting }\end{array}$ & 50 & $42 / 8$ & $\begin{array}{c}0.5 \mathrm{~g} \text { of powdered } \\
\text { ginger/cap, } 2 \\
\text { capsules/dose }+4 \mathrm{mg} \\
\text { of ondansetron (i.v.) }\end{array}$ & 50 & $38 / 12$ & $\begin{array}{c}2 \text { capsules of } \\
\text { placebo/dose }+4 \\
\mathrm{mg} \text { of ondansetron } \\
\text { (i.v.) }\end{array}$ & $\begin{array}{c}1 \mathrm{~h} \text { before } \\
\text { induction of } \\
\text { general anesthesia }\end{array}$ & Double-blind \\
\hline \multirow[t]{2}{*}{$\begin{array}{l}\text { Ozgoli et al. } \\
(2009) \text { [43] }\end{array}$} & Iran & $\begin{array}{l}\text { Randomized } \\
\text { controlled trial }\end{array}$ & $\begin{array}{c}\text { Primary } \\
\text { dysmenorrhea }\end{array}$ & 50 & $0 / 50$ & $\begin{array}{l}250 \mathrm{mg} \text { of powdered } \\
\text { ginger/cap, } 4 \\
\text { capsules/day }\end{array}$ & 50 & $0 / 50$ & $\begin{array}{c}250 \mathrm{mg} \text { of } \\
\text { mefenamic } \\
\text { acid/cap, } 4 \\
\text { capsules/day }\end{array}$ & 3 days & Double-blind \\
\hline & Iran & $\begin{array}{l}\text { Randomized } \\
\text { controlled trial }\end{array}$ & $\begin{array}{c}\text { Primary } \\
\text { dysmenorrhea }\end{array}$ & 50 & $0 / 50$ & $\begin{array}{l}250 \mathrm{mg} \text { of powdered } \\
\text { ginger/cap, } 4 \\
\text { capsules/day }\end{array}$ & 50 & $0 / 50$ & $\begin{array}{c}400 \mathrm{mg} \text { of } \\
\text { ibuprofen/cap, } 4 \\
\text { capsules/day }\end{array}$ & 3 days & Double-blind \\
\hline \multirow[t]{2}{*}{$\begin{array}{l}\text { Kashefi et al. } \\
(2013) \text { [44] }\end{array}$} & Iran & $\begin{array}{l}\text { Randomized } \\
\text { controlled trial }\end{array}$ & $\begin{array}{c}\text { Primary } \\
\text { dysmenorrhea }\end{array}$ & $\begin{array}{l}47 \text { (1st month); } \\
45 \text { (2nd month) }\end{array}$ & $\begin{array}{c}\text { 0/47 (1st month) } \\
0 / 45 \text { (2nd month) }\end{array}$ & $\begin{array}{l}250 \mathrm{mg} \text { of powdered } \\
\text { ginger/cap, } 3 \\
\text { capsules/day } \\
\end{array}$ & $\begin{array}{l}54 \text { (1st month); } \\
53 \text { (2nd month) }\end{array}$ & $\begin{array}{l}\text { 0/54 (1st month); } \\
0 / 53 \text { (2nd month) }\end{array}$ & $\begin{array}{l}220 \mathrm{mg} \text { of zinc } \\
\text { sulfate/cap, } 3 \\
\text { capsules/day }\end{array}$ & 4 days & N/A \\
\hline & Iran & $\begin{array}{l}\text { Randomized } \\
\text { controlled trial }\end{array}$ & $\begin{array}{c}\text { Primary } \\
\text { dysmenorrhea }\end{array}$ & $\begin{array}{l}47 \text { (1st month); } \\
45 \text { (2nd month) }\end{array}$ & $\begin{array}{c}\text { 0/47 (1st month) } \\
\text { 0/45 (2nd month) }\end{array}$ & $\begin{array}{l}250 \mathrm{mg} \text { of powdered } \\
\text { ginger/cap, } 3 \\
\text { capsules/day }\end{array}$ & $\begin{array}{l}45 \text { (1st month); } \\
42 \text { (2nd month) }\end{array}$ & $\begin{array}{l}\text { 0/45 (1st month); } \\
\text { 0/42 (2nd month) }\end{array}$ & $\begin{array}{l}220 \mathrm{mg} \text { of } \\
\text { lactose/cap, } 3 \\
\text { capsules/day }\end{array}$ & 4 days & $\mathrm{N} / \mathrm{A}$ \\
\hline \multirow[t]{2}{*}{$\begin{array}{l}\text { Rahnama et al. } \\
(2012)[45]\end{array}$} & Iran & $\begin{array}{l}\text { Randomized } \\
\text { controlled trial }\end{array}$ & $\begin{array}{c}\text { Primary } \\
\text { dysmenorrhea }\end{array}$ & 59 & $0 / 59$ & $\begin{array}{l}500 \mathrm{mg} \text { of powdered } \\
\text { ginger root/cap, } 3 \\
\text { capsules/day }\end{array}$ & 59 & $0 / 59$ & $\begin{array}{l}500 \mathrm{mg} \text { of toast } \\
\text { powder } \\
\text { (placebo)/cap, } 3 \\
\text { capsules/day }\end{array}$ & $\begin{array}{l}\text { From two days } \\
\text { before the onset of } \\
\text { menstrual period } \\
\text { to first three days } \\
\text { of menstrual } \\
\text { period }\end{array}$ & Double-blind \\
\hline & Iran & $\begin{array}{l}\text { Randomized } \\
\text { controlled trial }\end{array}$ & $\begin{array}{c}\text { Primary } \\
\text { dysmenorrhea }\end{array}$ & 59 & $0 / 59$ & $\begin{array}{l}500 \mathrm{mg} \text { of powdered } \\
\text { ginger root/cap, } 3 \\
\text { capsules/day }\end{array}$ & 46 & $0 / 46$ & $\begin{array}{l}500 \mathrm{mg} \text { of toast } \\
\text { powder } \\
\text { (placebo)/cap, } 3 \\
\text { capsules/day }\end{array}$ & $\begin{array}{l}\text { First three days of } \\
\text { the menstrual } \\
\text { period }\end{array}$ & Double-blind \\
\hline
\end{tabular}


Table 1. Cont.

\begin{tabular}{|c|c|c|c|c|c|c|c|c|c|c|c|}
\hline \multirow{2}{*}{ Author (Year) } & \multirow{2}{*}{$\begin{array}{c}\text { Cohort } \\
\text { Allocation }\end{array}$} & \multirow{2}{*}{ Study Design } & \multirow{2}{*}{$\begin{array}{c}\text { Type of } \\
\text { Disease/Symptom }\end{array}$} & \multicolumn{3}{|c|}{ Intervention } & \multicolumn{3}{|c|}{ Comparator } & \multirow{2}{*}{ Duration } & \multirow{2}{*}{ Blind } \\
\hline & & & & Number & M/F & Dosage & Number & $\mathbf{M} / \mathbf{F}$ & Dosage & & \\
\hline $\begin{array}{l}\text { Black et al. } \\
\text { (2010) [46] }\end{array}$ & $\begin{array}{l}\text { United } \\
\text { States }\end{array}$ & $\begin{array}{l}\text { Crossover } \\
\text { randomized } \\
\text { controlled trial }\end{array}$ & $\begin{array}{l}\text { Muscle pain, } \\
\text { inflammation, } \\
\text { and dysfunction } \\
\text { induced by } \\
\text { eccentric exercise }\end{array}$ & 27 & $12 / 15$ & $\begin{array}{l}\text { Six capsules } \\
\text { containing } 2 \mathrm{~g} \text { of dried } \\
\text { ginger extract with } \\
250 \mathrm{~mL} \text { of water } \\
\text { and one tablespoon of } \\
\text { olive oil. }\end{array}$ & 27 & $12 / 15$ & $\begin{array}{c}\text { Six capsules } \\
\text { containing } 2 \mathrm{~g} \text { of } \\
\text { flour with } 250 \mathrm{~mL} \\
\text { of water } \\
\text { and one } \\
\text { tablespoon of olive } \\
\text { oil. }\end{array}$ & Single-dose & Double-blind \\
\hline \multirow[t]{2}{*}{$\begin{array}{l}\text { Black et al. } \\
\text { (2009) [47] }\end{array}$} & $\begin{array}{l}\text { United } \\
\text { States }\end{array}$ & $\begin{array}{l}\text { Randomized } \\
\text { controlled trial }\end{array}$ & $\begin{array}{c}\text { Muscle pain } \\
\text { caused by } \\
\text { eccentric exercise }\end{array}$ & $\begin{array}{l}\text { Raw ginger } \\
\text { study: } 17\end{array}$ & $\begin{array}{c}\text { Raw ginger study: } \\
3 / 14\end{array}$ & $\begin{array}{l}\text { Raw ginger study: } 2 \mathrm{~g} \\
\text { of raw ginger } / \text { day }\end{array}$ & $\begin{array}{l}\text { Raw ginger } \\
\text { study: } 17\end{array}$ & $\underset{3 / 14}{\text { Raw ginger study: }}$ & $\begin{array}{l}\text { Raw ginger study: } \\
2 \text { g of yellow } \\
\text { cornflower/day }\end{array}$ & 11 days & Double-blind \\
\hline & $\begin{array}{l}\text { United } \\
\text { States }\end{array}$ & $\begin{array}{l}\text { Randomized } \\
\text { controlled trial }\end{array}$ & $\begin{array}{l}\text { Muscle pain } \\
\text { caused by } \\
\text { eccentric exercise }\end{array}$ & $\begin{array}{l}\text { Heat-treated } \\
\text { ginger study: } 20\end{array}$ & $\begin{array}{c}\text { Heat-treated } \\
\text { ginger study: } 7 / 13\end{array}$ & $\begin{array}{l}\text { Heat-treated ginger } \\
\text { study: } 2 \text { of of } \\
\text { heat-treated } \\
\text { ginger/day }\end{array}$ & $\begin{array}{l}\text { Heat-treated } \\
\text { ginger study: } 20\end{array}$ & $\begin{array}{l}\text { Heat-treated } \\
\text { ginger study: 7/13 }\end{array}$ & $\begin{array}{l}\text { Heat-treated } \\
\text { ginger study: } 2 \mathrm{~g} \\
\text { of powdered } \\
\text { brown sugar/day }\end{array}$ & 11 days & Double-blind \\
\hline $\begin{array}{l}\text { Mahluji et al. } \\
\text { (2013) [48] }\end{array}$ & Iran & $\begin{array}{l}\text { Randomized } \\
\text { controlled trial }\end{array}$ & $\begin{array}{l}\text { Type } 2 \text { diabetes } \\
\text { mellitus }\end{array}$ & 28 & $\mathrm{~N} / \mathrm{A}$ & $\begin{array}{c}1 \mathrm{~g} \text { of powdered } \\
\text { ginger/tab, } 2 \\
\text { tablets/day }\end{array}$ & 30 & $\mathrm{~N} / \mathrm{A}$ & $\begin{array}{l}1 \mathrm{~g} \text { of corn } \\
\text { starch/tab, } 2 \\
\text { tablets/day }\end{array}$ & 8 weeks & Double-blind \\
\hline $\begin{array}{l}\text { Khandouzi et al. } \\
\text { (2013) [49] }\end{array}$ & Iran & $\begin{array}{l}\text { Randomized } \\
\text { controlled trial }\end{array}$ & $\begin{array}{l}\text { Type } 2 \text { diabetes } \\
\text { mellitus }\end{array}$ & 22 & $5 / 17$ & $\begin{array}{l}1 \mathrm{~g} \text { of powdered } \\
\text { ginger/cap, } 2 \\
\text { capsules/day }\end{array}$ & 19 & 9/10 & Lactose (placebo) & 12 weeks & Double-blind \\
\hline $\begin{array}{l}\text { Phillips et al. } \\
\text { (1992) [50] }\end{array}$ & $\begin{array}{c}\text { United } \\
\text { Kingdom }\end{array}$ & $\begin{array}{c}\text { Crossover } \\
\text { randomized } \\
\text { controlled trial }\end{array}$ & Gastric emptying & 16 & $\mathrm{~N} / \mathrm{A}$ & $\begin{array}{l}500 \mathrm{mg} \text { of powdered } \\
\text { ginger/cap, } 2 \\
\text { capsules/dose } \\
\end{array}$ & 16 & N/A & $\begin{array}{c}500 \mathrm{mg} \text { of } \\
\text { lactose/cap, } 2 \\
\text { capsules/dose }\end{array}$ & Single-dose & Double-blind \\
\hline \multirow[t]{2}{*}{$\begin{array}{l}\text { Jiang et al. } \\
\text { (2013) [14] }\end{array}$} & $\begin{array}{l}\text { United } \\
\text { States }\end{array}$ & $\begin{array}{l}\text { Randomized } \\
\text { controlled trial }\end{array}$ & $\begin{array}{l}\text { Normal risk for } \\
\text { colorectal cancer }\end{array}$ & 14 & $\mathrm{~N} / \mathrm{A}$ & $\begin{array}{l}250 \mathrm{mg} \text { of ginger } \\
\text { extract/cap, } 8 \\
\text { capsules/day }\end{array}$ & 16 & N/A & Placebo (lactose) & 28 days & Double-blind \\
\hline & $\begin{array}{l}\text { United } \\
\text { States }\end{array}$ & $\begin{array}{l}\text { Randomized } \\
\text { controlled trial }\end{array}$ & $\begin{array}{l}\text { High risk for } \\
\text { colorectal cancer }\end{array}$ & 10 & $4 / 6$ & $\begin{array}{l}250 \mathrm{mg} \text { of ginger } \\
\text { extract/cap, } 8 \\
\text { capsules/day }\end{array}$ & 10 & $3 / 7$ & Placebo (lactose) & 28 days & Double-blind \\
\hline $\begin{array}{l}\text { Citronberg et al. } \\
\text { (2013) [51] }\end{array}$ & $\begin{array}{l}\text { United } \\
\text { States }\end{array}$ & $\begin{array}{l}\text { Randomized } \\
\text { controlled trial }\end{array}$ & $\begin{array}{c}\text { Cell cycle } \\
\text { biomarkers in the } \\
\text { normal-appearing } \\
\text { colonic mucosa of } \\
\text { patients at } \\
\text { increased risk for } \\
\text { colorectal cancer }\end{array}$ & 10 & $4 / 6$ & $\begin{array}{l}250 \mathrm{mg} \text { of ginger } \\
\text { extract powder (5\% } \\
\text { gingerols)/cap, } 8 \\
\text { capsules/day, bid }\end{array}$ & 10 & $3 / 7$ & $\begin{array}{c}250 \mathrm{mg} \text { of lactose } \\
\text { powder/cap, } 8 \\
\text { capsules/day, bid }\end{array}$ & 28 days & Double-blind \\
\hline $\begin{array}{l}\text { Zick et al. } \\
\text { (2011) [52] }\end{array}$ & $\begin{array}{l}\text { United } \\
\text { States }\end{array}$ & $\begin{array}{l}\text { Randomized } \\
\text { controlled trial }\end{array}$ & $\begin{array}{l}\text { Eicosanoids level } \\
\text { of patients with } \\
\text { normal risk for } \\
\text { colorectal cancer }\end{array}$ & 16 & $\mathrm{~N} / \mathrm{A}$ & $\begin{array}{l}250 \mathrm{mg} \text { of dry ginger } \\
\text { root extract/cap, } 8 \\
\text { capsules/day }\end{array}$ & 17 & N/A & $\begin{array}{c}250 \mathrm{mg} \text { of } \\
\text { lactose/cap, } 8 \\
\text { capsules/day }\end{array}$ & 28 days & Triple-blind \\
\hline
\end{tabular}


Table 1. Cont.

\begin{tabular}{|c|c|c|c|c|c|c|c|c|c|c|c|}
\hline \multirow{2}{*}{ Author (Year) } & \multirow{2}{*}{$\begin{array}{c}\text { Cohort } \\
\text { Allocation }\end{array}$} & \multirow{2}{*}{ Study Design } & \multirow{2}{*}{$\begin{array}{c}\text { Type of } \\
\text { Disease/Symptom }\end{array}$} & \multicolumn{3}{|c|}{ Intervention } & \multicolumn{3}{|c|}{ Comparator } & \multirow{2}{*}{ Duration } & \multirow{2}{*}{ Blind } \\
\hline & & & & Number & M/F & Dosage & Number & M/F & Dosage & & \\
\hline $\begin{array}{l}\text { Zick et al. } \\
\text { (2014) [53] }\end{array}$ & $\begin{array}{l}\text { United } \\
\text { States }\end{array}$ & $\begin{array}{l}\text { Randomized } \\
\text { controlled trial }\end{array}$ & $\begin{array}{l}\text { Eicosanoids level } \\
\text { of patients with } \\
\text { increased risk for } \\
\text { colorectal cancer }\end{array}$ & 10 & $4 / 6$ & $\begin{array}{c}250 \mathrm{mg} \text { of dry ginger } \\
\text { root extract (5\% } \\
\text { gingerols)/cap, } 8 \\
\text { capsules/day }\end{array}$ & 10 & $3 / 7$ & $\begin{array}{c}250 \mathrm{mg} \text { of } \\
\text { lactose/cap, } 8 \\
\text { capsules/day }\end{array}$ & 28 days & Double-blind \\
\hline \multirow[t]{2}{*}{$\begin{array}{l}\text { Tilburg et al. } \\
\text { (2014) [54] }\end{array}$} & $\begin{array}{l}\text { United } \\
\text { States }\end{array}$ & $\begin{array}{l}\text { Randomized } \\
\text { controlled trial }\end{array}$ & $\begin{array}{l}\text { Irritable bowel } \\
\text { syndrome }\end{array}$ & 15 & $\mathrm{~N} / \mathrm{A}$ & $\begin{array}{l}1 \mathrm{~g} \text { of ginger in } \\
\text { capsules }(2.29 \mathrm{mg} / \mathrm{g} \text { of } \\
\text { gingerols and } \\
\text { 6-shogaol) }\end{array}$ & 15 & $\mathrm{~N} / \mathrm{A}$ & $\begin{array}{l}\text { Brown sugar in } \\
\text { capsules }\end{array}$ & 28 days & Double-blind \\
\hline & $\begin{array}{l}\text { United } \\
\text { States }\end{array}$ & $\begin{array}{l}\text { Randomized } \\
\text { controlled trial }\end{array}$ & $\begin{array}{l}\text { Irritable bowel } \\
\text { syndrome }\end{array}$ & 15 & $\mathrm{~N} / \mathrm{A}$ & $\begin{array}{l}2 \mathrm{~g} \text { of ginger in } \\
\text { capsules }(2.29 \mathrm{mg} / \mathrm{g} \text { of } \\
\text { gingerols and } \\
\text { 6-shogaol) }\end{array}$ & & & & & \\
\hline $\begin{array}{l}\text { Wigler et al. } \\
\text { (2003) [55] }\end{array}$ & Israel & $\begin{array}{c}\text { Crossover } \\
\text { randomized } \\
\text { controlled trial }\end{array}$ & $\begin{array}{l}\text { Symptomatic } \\
\text { gonarthritis }\end{array}$ & $\begin{array}{l}\text { Group } 1 \text { (ginger } \\
\text { first): 14; Group } \\
2 \text { (placebo first): } \\
15\end{array}$ & $\begin{array}{l}\text { Group } 1 \text { (ginger } \\
\text { first): } 1 / 13 \text { Group } 2 \\
\text { (placebo first): } 5 / 10\end{array}$ & $\begin{array}{c}250 \mathrm{mg} \text { of ginger } \\
\text { extract (10 mg of } \\
\text { gingerol)/cap, } 4 \\
\text { capsules/day }\end{array}$ & $\begin{array}{c}\text { Group } 2 \text { (placebo } \\
\text { first): } 15 ; \text { Group } \\
1 \text { (ginger first): } \\
14\end{array}$ & $\begin{array}{l}\text { Group } 2 \text { (placebo } \\
\text { first): } 5 / 10 ; \text { Group1 } \\
\text { (ginger first): } 1 / 13\end{array}$ & $\begin{array}{c}\text { Placebo capsules, } 4 \\
\text { capsules/day }\end{array}$ & $\begin{array}{l}\text { Two periods of } 12 \\
\text { weeks }\end{array}$ & Double-blind \\
\hline
\end{tabular}

* DMA regimen: dexamethasone, metoclopramide, and aprepitant; M/F: Male/Female; N/A: Not available. 
(a)

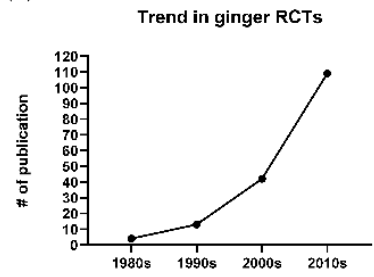

(d)

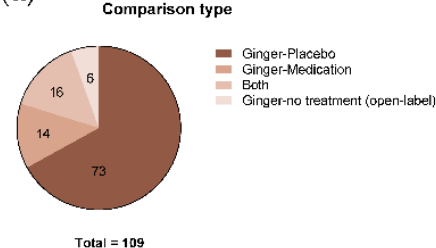

(b)

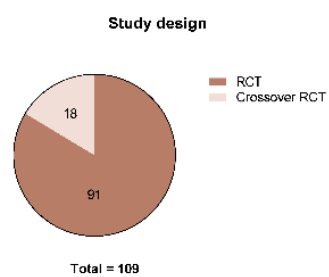

(e)

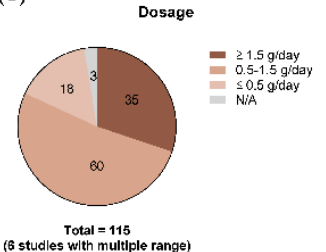

(c)

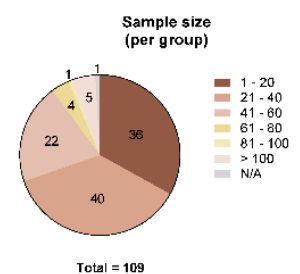

(f)

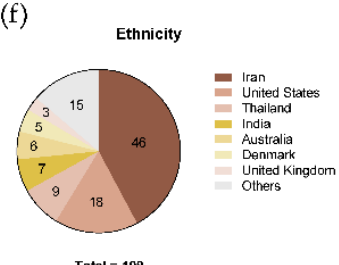

Figure 2. Features of ginger Randomized controlled trial (RCT) characteristics. (a) The trend in the publication of ginger RCTs over the decades, (b) the types of study design, (c) the ranges of pooled sample size per group, (d) the types of comparison in intervention, (e) the ranges of adopted dosage, and (f) the variety of ethnicity. N/A: Not available.

\subsection{Clinical Effects of Ginger}

The effects of ginger were reported in a variety of diseases and health conditions. In the following sections, we cover the five significant biological effects of ginger that were mainly examined in the included studies. In addition, other potential effects are also briefly summarized and discussed. Table 2 presents the key finding of each study introduced in Table 1. The key finding of other publications is provided in Table S3.

\subsubsection{Antiemetic Function}

Major clinical trials with ginger were performed to evaluate its antiemetic activity $(n=47)$. Among these, CINV, a phenomenon induced by chemotherapeutic agents and which activates neurotransmitters as a side effect, was the most frequently investigated subject $(n=16)$. From 16 trials, eight demonstrated the positive effect of ginger treatment on the prevention and alleviation of CINV. Sanaati et al. reported that ginger significantly improved the quality of life in CINV group of patients who had received the first cycle of moderately to highly emetogenic chemotherapy compared to the placebo (median (interquartile range) $=124.5(113.2,126)$ vs. $111(99,126) ; p=0.043)$ [18]. Furthermore, ginger effectively reduced acute and delayed CINV in both children and adults [56,57]. On the other hand, Thamlikitkul et al. and Li et al. concluded that ginger showed an insufficient effect on the prophylaxis of acute and delayed nausea and vomiting induced by an Adriamycin-cyclophosphamide regimen and a cisplatin regimen, respectively, which are highly emetogenic regimens $[19,20]$.

Nausea and vomiting of pregnancy (NVP), also called hyperemesis gravidarum in severe cases, is a symptom that commonly occurs in pregnant women and has the potential to cause nutritional deficiency. There were 14 clinical trials regarding the alleviative effect of ginger on NVP. Eight studies investigated the antiemetic effect of ginger by comparing it to a placebo group, revealing significant effects in the ginger treatment group. Simultaneously, ginger showed a similar effect compared to other medication groups, such as vitamin B6 (pyridoxine), antihistamine, or metoclopramide. However, a study by Ensiyeh et al. concluded that ginger is more effective in relieving the severity of nausea compared to vitamin B6 ( $p=0.024)$ [3]. 
Table 2. Evaluation system and key finding of the included studies with a "high quality of evidence" (quality assessment score is of at least 10).

\begin{tabular}{|c|c|c|c|}
\hline Author (Year) & Evaluation Outcome System & Main Result & Adverse Effect \\
\hline Marx et al. (2017) [17] & FLIE-5DR questionnaire, RINVR & $\begin{array}{l}\text { Compared with placebo, ginger supplementation therapy } \\
\text { can improve the chemotherapy-induced nausea-related } \\
\text { quality of life and relieve vomiting and fatigue caused by } \\
\text { chemotherapy. }\end{array}$ & No \\
\hline Sanaati et al. (2016) [18] & $\begin{array}{l}\text { Effects of the groups on nausea and vomiting using the } \\
\text { generalized estimating equations (GEE) model }\end{array}$ & $\begin{array}{l}\text { Ginger treatment reduced the frequency of vomiting and } \\
\text { nausea significantly. }\end{array}$ & No \\
\hline Thamlikitkul et al. (2016) [19] & $\begin{array}{l}\text { Nausea score ( by VAS), vomiting incidence, rate of rescue } \\
\text { medication use, and incidence of chemotherapy dose reduction }\end{array}$ & $\begin{array}{l}\text { This study indicated that taking } 1 \mathrm{~g} \text { of ginger for five days } \\
\text { from the first day of chemotherapy had no effect in reducing } \\
\text { the nausea severity of breast cancer patients receiving } \\
\text { Adriamycin and cyclophosphamide chemotherapy. }\end{array}$ & No \\
\hline Li et al. (2017) [20] & Incidence and severity of CINV by the MASCC Antiemesis Tool & $\begin{array}{l}\text { In lung cancer patients who received cisplatin regimen, } \\
\text { taking ginger as an adjuvant drug for antiemetics was } \\
\text { ineffective in reducing the incidence and severity of CINV. }\end{array}$ & No \\
\hline Ansari et al. (2016) [21] & $\begin{array}{l}\text { Episodes of vomiting and nausea severity by nausea and } \\
\text { vomiting grading }\end{array}$ & $\begin{array}{l}\text { There was no significant improvement in breast cancer } \\
\text { patients receiving chemotherapy regime-induced CINV } \\
\text { upon ginger treatment. Thus, additional study is needed to } \\
\text { make a conclusion. }\end{array}$ & No \\
\hline Sharifzadeh et al. (2018) [4] & Rhodes questionnaire 2 & $\begin{array}{l}\text { In relieving moderate to mild nausea and vomiting caused } \\
\text { by pregnancy, ginger groups had similar effects to vitamin } \\
\text { B6 and were more effective than the placebo group. }\end{array}$ & No \\
\hline Matsumura et al. (2015) [22] & $\begin{array}{l}\text { Creatine kinase, lactate dehydrogenase, } 1 \mathrm{RM} \text {, muscle soreness } \\
\text { (by VAS), Circumference, ROM-flexion, ROM-extension, skin } \\
\text { temp-non-dominant arm, skin temp-dominant arm }\end{array}$ & $\begin{array}{l}\text { This study showed that taking } 4 \mathrm{~g} \text { of ginger may promote } \\
\text { the recovery of muscle strength after intense exercise but } \\
\text { has no effect on indicators of muscle damage or delayed } \\
\text { onset muscle soreness. }\end{array}$ & No \\
\hline Martins et al. (2018) [23] & $\begin{array}{l}\text { Four-point scale, faces pain scale, visual numeric scale, nausea, } \\
\text { ordinal scale, photophobia, phonophobia, and treatment } \\
\text { satisfaction }\end{array}$ & $\begin{array}{l}\text { The ginger-treated group showed a significant effect in } \\
\text { reducing migraine attacks. }\end{array}$ & No \\
\hline Arzati et al. (2017) [24] & $\begin{array}{l}\text { FBS, total cholesterol, TG, LDL-cholesterol, HDL-cholesterol, } \\
\text { HbA1C }\end{array}$ & $\begin{array}{l}\text { Ginger supplementation significantly lowered fasting blood } \\
\text { sugar, the mean variation of } \mathrm{HbA1C} \text {, and LDL/HDL ratio. }\end{array}$ & No \\
\hline Attari et al. (2016) [25] & Glucose, leptin, resistin, adiponectin, insulin, HOMA-IR, QUICKI & $\begin{array}{l}\text { A little beneficial effect of ginger powder supplementation } \\
\text { was found regarding improving biochemical obesity } \\
\text { indicator and weight loss. }\end{array}$ & No \\
\hline
\end{tabular}


Table 2. Cont

\begin{tabular}{|c|c|c|c|}
\hline Author (Year) & Evaluation Outcome System & Main Result & Adverse Effect \\
\hline Attari et al. (2015) [26] & Obesity-associated parameters $^{1}$ & $\begin{array}{l}\text { Subjects with the } A A, U C P 1 \text {, and Trp64Trp genotypes of } \\
\beta 3 \text { ADR had significantly decreased anthropometric } \\
\text { measurements and total appetite scores compared with the } \\
\text { placebo group, but other evaluation outcomes were not } \\
\text { significant. }\end{array}$ & No \\
\hline $\begin{array}{l}\text { Mozaffari-Khosravi et al. } \\
\text { (2016) [13] }\end{array}$ & Serum TNF- $\alpha$, serum IL- $1 \beta$ & $\begin{array}{l}\text { In knee osteoarthritis patients, serum TNF- } \alpha \text { NF-m IL- } 1 \beta \\
\text { was decreased in both groups, with a lower level in the } \\
\text { ginger group than the placebo group. }\end{array}$ & No \\
\hline Kashefi et al. (2015) [28] & Percentage by which the mean hemorrhage decreased (\%) & $\begin{array}{l}\text { Ginger treatment reduced menstrual blood loss } \\
\text { significantly during three interventions. }\end{array}$ & No \\
\hline Paritakul et al. (2016) [29] & $\begin{array}{l}\text { Breast milk volume on day three, breast milk volume on day } \\
\text { seven, and serum prolactin level }\end{array}$ & $\begin{array}{l}\text { Ginger treatment significantly increased milk volume on } \\
\text { the third day compared to the placebo group. However, no } \\
\text { significant difference was found in the milk volume and } \\
\text { serum prolactin levels on the seventh day between the } \\
\text { ginger and placebo groups. }\end{array}$ & No \\
\hline Ryan et al. (2012) [30] & $\begin{array}{l}\text { Seven point semantic rating, } 13 \text { item symptom inventory, } \\
\text { and functional assessment of chronic illness therapy general }\end{array}$ & $\begin{array}{l}\text { In adult cancer patients, a daily dose of } 0.5-1.0 \mathrm{~g} \text { of ginger } \\
\text { was helpful in relieving the severity of acute } \\
\text { chemotherapy-induced nausea }\end{array}$ & Yes \\
\hline Zick et al. (2008) [31] & $\begin{array}{l}\text { Prevalence and severity of delayed nausea and vomiting (by } \\
\text { Morrow Assessment of Nausea and Emesis questionnaire) }\end{array}$ & $\begin{array}{l}\text { Taking ginger as a reduction of CINV was insufficient, } \\
\text { and there was no additional benefit for reducing the } \\
\text { severity of acute and delayed CINV. }\end{array}$ & No \\
\hline Fahimi et al. (2010) [32] & $\begin{array}{l}\text { Prevalence, severity, and duration of acute and delayed nausea } \\
\text { and vomiting (by Morrow Assessment of Nausea and Emesis) }\end{array}$ & $\begin{array}{l}\text { Ginger treatment showed no effect in reducing the } \\
\text { prevalence, severity, and duration of both acute and } \\
\text { delayed nausea and vomiting }\end{array}$ & No \\
\hline Yekta et al. (2012) [33] & $\begin{array}{l}\text { Self-made, two-part self-reporting instrument (number of } \\
\text { vomiting, use of other antiemetics, side effects) }\end{array}$ & $\begin{array}{l}\text { The ginger treatment group decreased vomiting at } \\
\text { anticipatory, acute, and delayed phases of patients who } \\
\text { received chemotherapy. }\end{array}$ & Yes \\
\hline
\end{tabular}


Table 2. Cont

\begin{tabular}{|c|c|c|c|}
\hline Author (Year) & Evaluation Outcome System & Main Result & Adverse Effect \\
\hline Ensiyeh et al. (2009) [3] & $\begin{array}{l}\text { Nausea scores, average number of vomiting episodes, follow-up } \\
\text { visit, a five-point Likert scale }\end{array}$ & $\begin{array}{l}\text { In early pregnancy, ginger intake had a stronger effect on } \\
\text { relieving the severity of nausea than vitamin B6 intake. } \\
\text { However, it had no significant difference in decreasing the } \\
\text { number of vomiting episodes. }\end{array}$ & No \\
\hline $\begin{array}{l}\text { Willetts et al. } \\
\text { (2003) [34] }\end{array}$ & Rhodes index of nausea, vomiting, and retching & $\begin{array}{l}\text { Regarding nausea experience and retching, the ginger } \\
\text { group was significantly lower than the placebo group of } \\
\text { pregnancy-induced nausea. }\end{array}$ & No \\
\hline Vutyavanich et al. (2001) [35] & $\begin{array}{l}\text { Nausea (VAS score), number of vomiting episodes, } \\
\text { and symptoms assessed by Likert scales }\end{array}$ & $\begin{array}{l}\text { Nausea and vomiting induced in pregnancy could be } \\
\text { relieved by ginger. }\end{array}$ & No \\
\hline $\begin{array}{l}\text { Fischer-Rasmussen et al. } \\
\text { (1990) [36] }\end{array}$ & $\begin{array}{l}\text { The severity of hyperemesis (by degree of nausea, vomiting, } \\
\text { and weight loss) }\end{array}$ & $\begin{array}{l}\text { Ginger treatment showed significantly greater relief on } \\
\text { hyperemesis than a placebo. }\end{array}$ & No \\
\hline Smith et al. (2004) [37] & $\begin{array}{l}\text { Incidence of nausea, dry retching, and vomiting (by Rhodes } \\
\text { index of nausea and vomiting form) and health status (by MOS } \\
36 \text { short form health survey) }\end{array}$ & $\begin{array}{l}\text { Pregnant women with nausea, dry retching, vomiting can } \\
\text { use ginger in the early stage of pregnancy to relieve the } \\
\text { severity of symptoms as effectively as vitamin B6 }\end{array}$ & No \\
\hline Biswas et al. (2011) [38] & $\begin{array}{l}\text { The severity of dysmenorrhea, nausea, and vomiting (by VAS), } \\
\text { average of nausea spells per day, vomiting episodes, average of } \\
\text { nausea episodes, and average number of vomiting in last week }\end{array}$ & $\begin{array}{l}\text { A ginger extract can be considered as safe therapy and } \\
\text { effective alternative for the reduction of nausea and } \\
\text { vomiting with no severe or serious adverse events. }\end{array}$ & No \\
\hline Firouzbakht et al. (2014) [39] & $\begin{array}{l}\text { The severity of nausea and the frequency of vomiting (by Likert } \\
\text { scale and VAS) }\end{array}$ & $\begin{array}{l}\text { After one week, the severity of nausea and vomiting was } \\
\text { reduced dramatically in } 60.6 \%, 42.7 \% \text { and } 61 \% \text { of the ginger, } \\
\text { placebo, and B6 groups, respectively. }\end{array}$ & Yes \\
\hline Arfeen et al. (1995) [40] & The incidence of PONV and the distribution of nausea score & $\begin{array}{l}0.5 \text { or } 1.0 \mathrm{~g} \text { of ginger showed no efficacy on the incidence by } \\
\text { postoperative nausea and vomiting. }\end{array}$ & No \\
\hline Eberhart et al. (2003) [41] & $\begin{array}{l}\text { The incidence rate of PONV, nausea, vomiting, and rescue } \\
\text { antiemetics }\end{array}$ & $\begin{array}{l}\text { There was no reduction in nausea, vomiting and the } \\
\text { demand for antiemetic rescue treatment in three groups. }\end{array}$ & Yes \\
\hline Mandal et al. (2014) [42] & $\begin{array}{l}\text { Episodes of nausea, retching, vomiting and rescue antiemetic (by } \\
\text { the score of Bellville), and severity of PONV (by VAS) }\end{array}$ & $\begin{array}{l}\text { Ginger combined with ondansetron may be more helpful in } \\
\text { controlled PONV than ondansetron alone. }\end{array}$ & No \\
\hline Ozgoli et al. (2009) [43] & Self-administered questionnaire & $\begin{array}{l}\text { When alleviating pain in women with primary } \\
\text { dysmenorrhea, ginger was comparable to mefenamic acid } \\
\text { and ibuprofen. }\end{array}$ & No \\
\hline
\end{tabular}


Table 2. Cont.

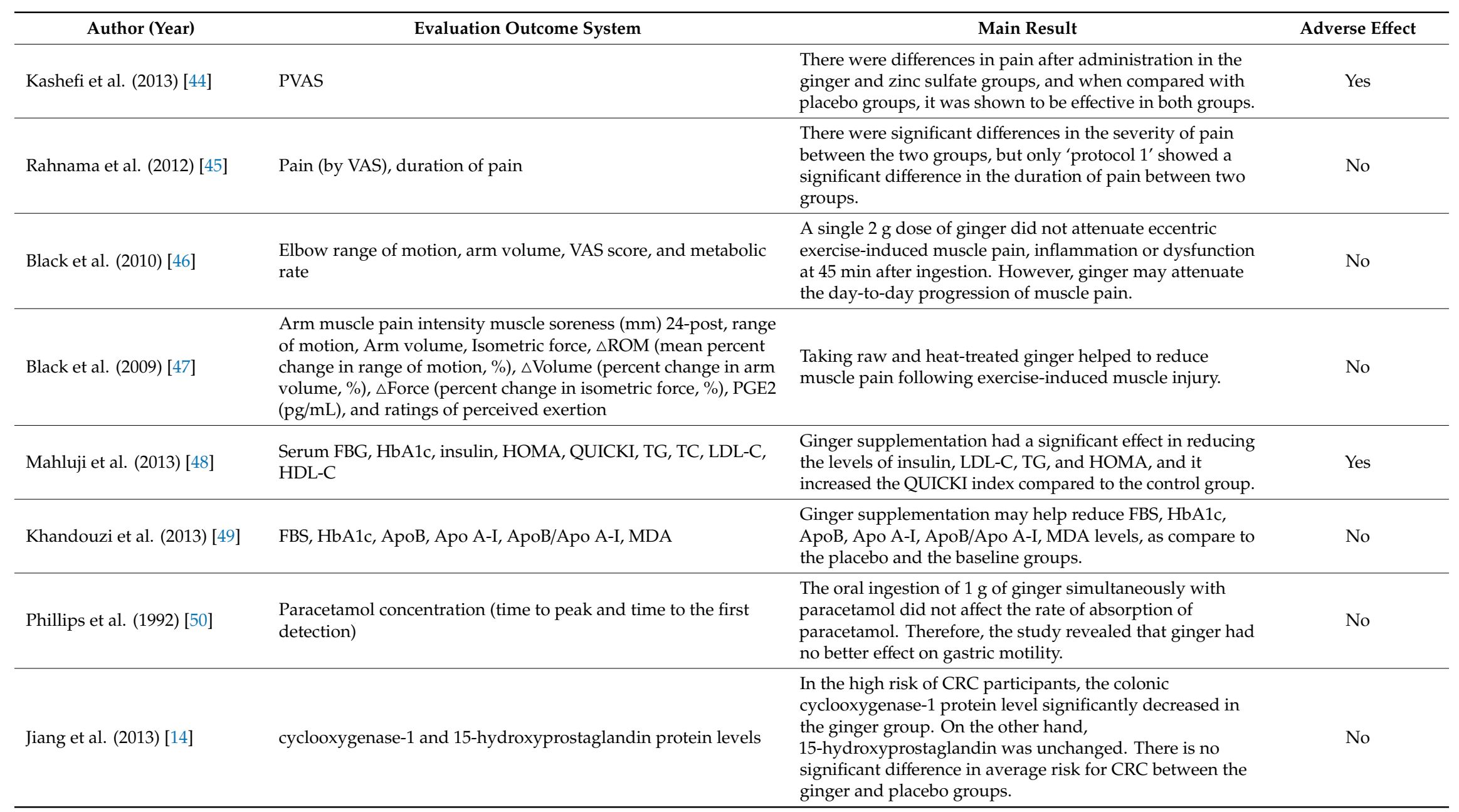


Table 2. Cont.

\begin{tabular}{|c|c|c|c|}
\hline Author (Year) & Evaluation Outcome System & Main Result & Adverse Effect \\
\hline Citronberg et al. (2013) [51] & $\begin{array}{l}\text { Bax, Bcl-2, p21, hTERT, MIB-1, Bax/Bcl-2 Ratio, Bax/hTERT Ratio, } \\
\text { Bax/MIB-1 Ratio, p21/hTERT Ratio, p21/MIB-1 Ratio, cell cycle } \\
\text { score (w/MIB-1), and cell cycle score (w/hTERT) }\end{array}$ & $\begin{array}{l}\text { Two grams of ginger extract may help in reducing the } \\
\text { proliferation of normal-appearing colorectal epithelium, as } \\
\text { well as increased apoptosis and differentiation relative to } \\
\text { proliferation—especially in the differentiation zone of } \\
\text { crypts }\end{array}$ & Yes \\
\hline Zick et al. (2011) [52] & $\begin{array}{l}\text { PGE2, 13-hydroxy-octadecadienoic acid, and 5-, 12-, and } 15 \\
\text { hydroxyeicosatetraenoic acid }\end{array}$ & $\begin{array}{l}\text { Ginger treatment may help to reduce eicosanoid levels by } \\
\text { inhibiting synthesis from arachidonic acid. Additionally, } \\
\text { ginger is considered to be safe for people with a high risk of } \\
\text { colorectal cancer. }\end{array}$ & No \\
\hline Zick et al. (2014) [53] & $\begin{array}{l}\text { PGE2, LTB4, 13-hydroxy-octadecadienoic acids, and 5-, 12-, } \\
\text { and 15-hydroxy-eicosatetraenoic acid }\end{array}$ & $\begin{array}{l}\text { Treating root extraction with ginger for people at high risk } \\
\text { for CRC for } 28 \text { days significantly decreased risk in the } \\
\text { normal colonic mucosa of arachidonic acid and significantly } \\
\text { increased LTB4, but other eicosanoids were ineffective. }\end{array}$ & Yes \\
\hline Tilburg et al. (2014) [54] & $\begin{array}{l}\text { Irritable bowel syndrome severity scale and adequate relief } \\
\text { rating scale }\end{array}$ & $\begin{array}{l}\text { In treating irritable bowel syndrome, ginger may not be a } \\
\text { proper choice because the result of study could not suggest } \\
\text { evidence for the better performance of the ginger treatment. }\end{array}$ & Yes \\
\hline Wigler et al. (2003) [55] & VAS of pain on movement and handicap & $\begin{array}{l}\text { After the crossover (three months), the ginger treatment } \\
\text { group showed a significantly higher effect compared to the } \\
\text { placebo group. }\end{array}$ & Yes \\
\hline \multicolumn{4}{|c|}{$\begin{array}{l}\text { 1: Body weight, BMI, waist circumference, hip circumference, waist-to-hip ratio, waist-to-height-ratio, body fat, body fat mass, fat-free body mass, appetite total score. CINV } \\
\text { chemotherapy-induced nausea and vomiting; FBS: fasting blood sugar; FLIE-5DR: functional living index emesis 5-day recall; HOMA-IR: homeostasis model assessment of insuli } \\
\text { resistance; HDL-C: high-density lipoprotein-cholesterol; HbA1c: Hemoglobin A1c; IL: interleukin; LDL-C: low-density lipoprotein-cholesterol; MDA: malondialdehyde; PVAS: pair } \\
\text { visual analog scale; QUICKI: quantitative insulin sensitivity check index; RINVR: Rhodes inventory of nausea, vomiting and retching; ROM: range of motion; TNF- } \alpha \text { : tumor necrosi } \\
\text { factor-alpha; VAS: visual analog scale; MASCC: multinational association of supportive care in cancer; Apo: apolipoprotein; TC: total cholesterol; TG: triglyceride; LTB4: leukotriene B4 } \\
\text { PG: prostaglandins treatment. }\end{array}$} \\
\hline
\end{tabular}


Postoperative nausea and vomiting (PONV) is an emetic event that is induced in the patients after surgical procedures, and it is mainly caused by the anesthetic. Ginger treatment was used as an intervention in eleven RCTs. However, five RCTs concluded that there were no significant results with ginger.

Still, ginger may have a beneficial effect in gynecological patients, according to the results from Apariman et al., Chaiyakunapruk et al., Phillips et al., and Bone et al. [58-61]. Finally, in the study by Dabaghzadeh et al., the researchers primally examined the effect of ginger and demonstrated its benefit on the prevention of nausea and vomiting induced by an antiretroviral regimen $(p=0.001)$ [62]. Efficacy on motion sickness and vertigo was also examined in several studies, with results indicating different aspects between the studies.

\subsubsection{Gastrointestinal Function}

As an extension of the antiemetic property, ginger has been studied for its protective effect on the gastrointestinal system. Seven RCTs examined ginger's effect on gastric function, mostly regarding gastric emptying and dysrhythmia. All studies that observed gastric emptying rate reported ginger as a digestive enhancer, except the study from Phillips et al., where they denied the facilitation of gastric function as demonstrated by the paracetamol absorption rate [50]. Lien et al. reported that ginger treatment significantly reduced tachygastric activity induced by circular vection, a phenomenon of feeling a rotating sensation without actual movement, in a rotating drum $(p<0.05)$. Gonlachanvit et al. investigated the beneficial effect of the ginger root in the prevention of slow-wave dysrhythmias induced by acute hyperglycemic events $(p<0.05)[63,64]$.

Four RCTs examined the anticancer effect of ginger, all of which evaluated the risk of colorectal cancer according to the ginger treatment. Collectively, ginger has a beneficial effect on colorectal cancer by reducing tumorigenic risk factors. However, Jiang et al. reported that participants with an average risk of colorectal cancer showed no significant aspect between the ginger and placebo groups [14]. Citronberg et al. investigated cell cycle markers with biopsies from the patients with increased risk of colorectal cancer and demonstrated the regulation of apoptotic and differentiation markers by ginger supplementation [51]. Lastly, one study from Miranda et al. examined the symptomatic relief on irritable bowel syndrome patients upon ginger application and found no evidence in the reduction of symptoms $(p>0.05)$ [54].

\subsubsection{Analgesic Function}

Seven RCTs examined the effect of ginger on primary dysmenorrhea. Four trials compared the analgesic effect with other medications such as mefenamic acid, ibuprofen, and zinc sulfate, which displayed similar efficiencies to ginger. Three trials adopted a placebo as a control group, reporting the reduction of pain by the level of visual analog scale. For example, Rahnama et al. reported that ginger significantly improved primary dysmenorrhea in ginger-treated patients for five days, beginning two days before the onset of menstruation [45]. Only one study concluded that ginger was an insufficient pain reliever upon comparison with stretching and exercising for alleviation [65].

Four RCTs administered ginger to a group of participants with muscular pain, with varied result observed. Two studies reported a lack of evidence regarding the effect of ginger, and the other two reported that ginger partially attenuated muscular pain compared to the placebo group. Migraines and headaches were examined to assess the pain-relieving attributes of ginger in three RCTs. Maghbooli et al. and Martins et al. compared the effects of sumatriptan and placebo, respectively, both showing there was a significant difference in symptom attenuation $(p<0.05)[23,66]$. Patients with low back pain and chest pain caused by percutaneous transluminal coronary angioplasty were also selected to assess the analgesic effects of ginger, and both studies concluded that ginger was a useful option for pain relief. 


\subsubsection{Inflammatory Effect}

Overall, eight RCTs reported the anti-inflammatory effect of ginger supplementation. Among them, arthritis-related diseases were the most conducted studies, particularly osteoarthritis (OA). Regarding OA, six studies investigated the efficiency of the constituents of ginger that serve as anti-inflammatory agents. All studies reported improvement following ginger intake compared to the control group. For instance, Mozaffari-Khosravi et al. proposed that benefits of ginger were observed due to a reduction in the level of the proinflammatory cytokines after three months of consuming $500 \mathrm{mg}$ of ginger powder [13]. Other studies showed a promising benefit of ginger in relieving pain in OA patients. Furthermore, no significant adverse effects were observed during the trials. An additional study that assessed ginger's effects on rheumatoid arthritis demonstrated improvement by reducing symptoms via inducing FOXP3 gene expression. Finally, Kulkarni et al. reported that ginger supplementation alone and combined with antitubercular treatment significantly helped to decrease tumor necrosis factor (TNF) alpha, ferritin, and malondialdehyde (MDA) levels compared to the control group [67].

\subsubsection{Metabolic Improvement}

Studies evaluating the efficiency of ginger in metabolic syndromes have also been widely conducted. Most of the included studies assessed the association of type 2 diabetes mellitus (T2DM) and obesity with ginger supplementation. In detail, five studies explored the effect of ginger on the diabetes-related indices such as glycemic markers, lipid level, and blood pressure, while four studies focused on various conditions related to obesity such as cardiovascular disease, serum adipocytokines, and breast cancer. For example, three studies evaluated the influence of ginger on biochemical parameters related to T2DM and demonstrated the significant lowering of fasting blood sugar, hemoglobin A1c ( $\mathrm{HbA1c})$, insulin sensitivity, and insulin resistance. Furthermore, lipid profile, inflammatory markers, and antioxidants were also affected by ginger intake, which was demonstrated by the reduction of the C-reactive protein, triglycerides (TG), low-density lipoprotein cholesterol (LDL-C), and malondialdehyde. Another study assessed the relationship between ginger and blood pressure in T2DM but showed no significant differences compared to the control group. Regarding obesity, the included study targeted the effect of ginger on obesity. In obese women, Attari et al. reported that ginger supplements had a minor benefit on weight loss, the reduction of insulin and homeostasis model assessment of insulin resistance (HOMA-IR), and the increasing of quantitative insulin sensitivity check index (QUICKI) [25]. In terms of obesity-related cardiovascular risk factors, ginger was reported to be beneficial in lowering the risk factors, such as body fat mass, body fat percentage, total cholesterol, waist circumference, waist-to-hip ratio, and insulin resistance. In addition, ginger was suggested to have antioxidant and anti-dysmetabolic effects in obese women with breast cancer. Finally, other studies aimed to explore the effect of ginger on lipid metabolism including fat utilization and triglyceride-lowering efficacy. In general, ginger was believed to provide potential benefits by reducing the risk factors of metabolic syndromes. Moreover, no serious adverse effects were observed in all included studies.

\subsubsection{Other Clinical Functions}

Besides the effects introduced above, several different functions, such as thermoregulatory, thrombotic, and respiratory function, were evaluated at the clinical level. The thermogenetic function of ginger was examined by three randomized crossover trials, and only one study observed the expected outcome. In terms of respiratory function, acute respiratory distress syndrome (ARDS) and asthma were examined to evaluate the improvement of symptoms. Ginger effectively reduced the duration of mechanical ventilation and the length of stay in the intensive care unit in ARDS patients; it also improved asthmatic symptoms. There were three studies regarding thrombotic function, and two studies reported that ginger had little effect on the thrombotic reaction. However, Bordia et al. reported 
that a single dose of $10 \mathrm{~g}$ of powdered ginger significantly reduced Adenosine diphosphate-induced and epinephrine-induced platelet aggregation in patients who were recovering from myocardial infarction [68]. Kashefi et al. administered ginger to 15-18 years old patients with heavy menstrual bleeding, and the ginger treatment group demonstrated a significant reduction in menstrual blood loss $(p<0.001)$ [28]. Paritakul et al. examined the effect of ginger on breast milk production in a group of women after delivery and concluded that ginger treatment significantly increased milk volume on the third day postpartum compared to the placebo $(p<0.01)$ [29].

\subsection{Adverse Effects}

Seventeen studies provided information about adverse effects in their research papers, most of which were not considered to be severely harmful to the participants. Among the adverse effects, gastrointestinal-related symptoms were mostly reported to reverse the gastrointestinal protective effect of ginger in other aspects. Heartburn, a general symptom of gastroesophageal reflux disease, was reported in sixteen studies. Five studies reported nausea as a side effect of ginger treatment, which was the primary topic evaluated to observe the clinical effect of ginger. Diarrhea was reported in two studies in groups of patients with heavy menstrual bleeding and after elective cesarean section. Other GI symptoms included abdominal pain, bloating, gas, and epigastric distress. Furthermore, cardiovascular symptoms and respiratory symptoms were observed in a ginger-treated patient group who underwent laparoscopic surgery. The types of adverse effects, incidence rate, and dosage are described in Table S4.

\subsection{Quality Assessment}

Firstly, regarding selection bias, three studies were judged as having a high risk of bias in random sequence generation, and 50 studies that were assigned to low risk described the methodological procedure of randomization. Thirty-six studies described the allocation concealment method with detailed explanations, and ten studies that provided ambiguous information about allocation were assigned to having an unclear risk. Sixty-three were judged as having a high risk of bias in allocation concealment. For example, in the study of Shirvani et al., participants were divided into a ginger treatment group and an exercising group, meaning that the allocation was easily predictable [65]. Second, in an aspect of performance bias, blinding was a common practice in more than $75 \%$ of the studies $(n=85)$. Eighteen studies were judged as showing an unclear risk, as they did not mention blinding or similarity of the administration appearance. The majority of studies did not report about the blinding of outcome assessment and were assigned to have a high risk in detection bias $(n=75$, $69.7 \%$ ). Thirty-one papers with low risk on this item described the blinding of the data analyzer or stated that their study design was triple-blinded. Attrition bias and reporting bias were the two least potential biases that could interrupt the results of the included trials, where 101 and 103 studies were assigned to low risks of attrition and reporting bias, respectively. Finally, 42 studies described the limitations of their studies and implied the possibility of any other biases, whereas 58 studies did not mention limitations and were assigned as a high risk. For better visualization, a methodological quality graph is introduced in Figure 3. 


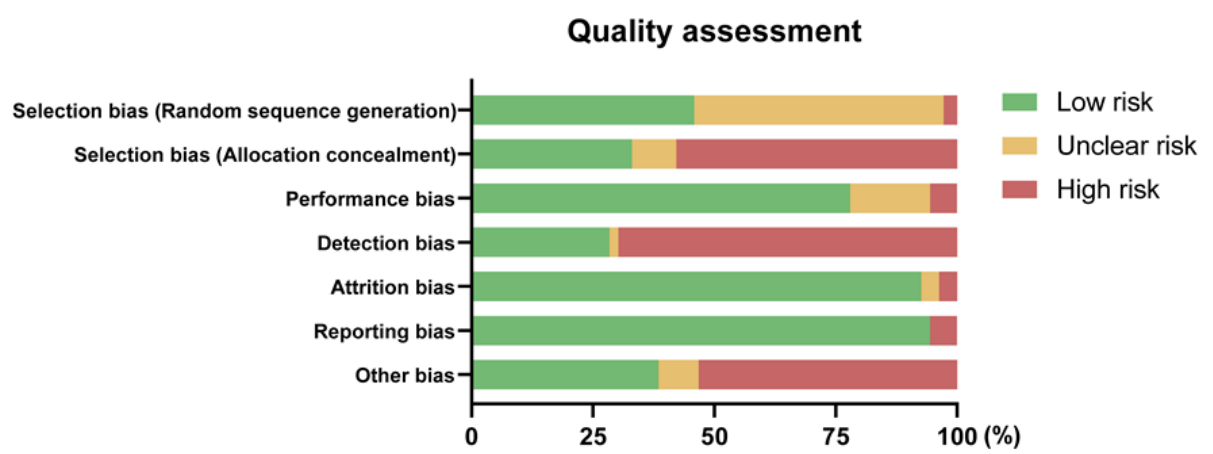

Figure 3. Methodological quality graph: The risk of bias for each item is expressed by percentage.

In addition to investigating each quality assessment item for overall studies, we also compared the quality of each important function based on our quality assessment scoring system, as described in Figure 4. The quality assessment (QA) outcomes and the QA score for each study are provided in Table S5.

(a)

QA score distribution (109 RCTs)

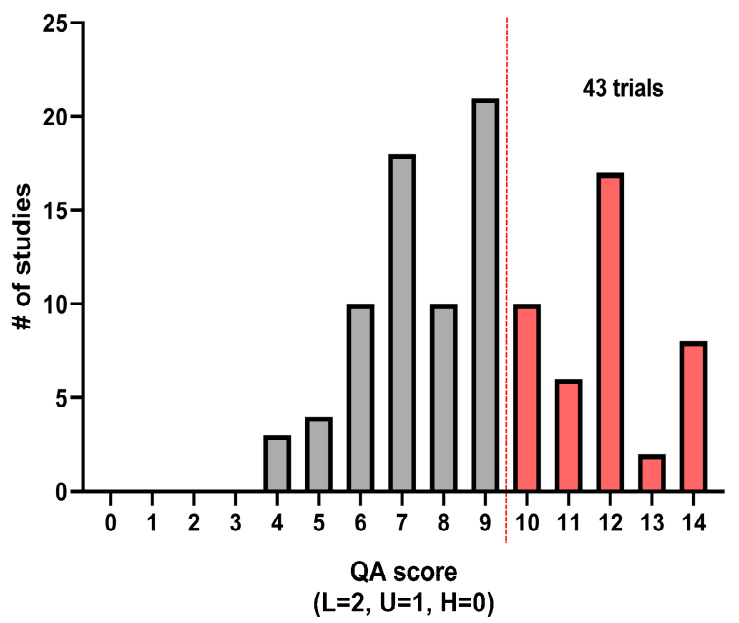

(b)
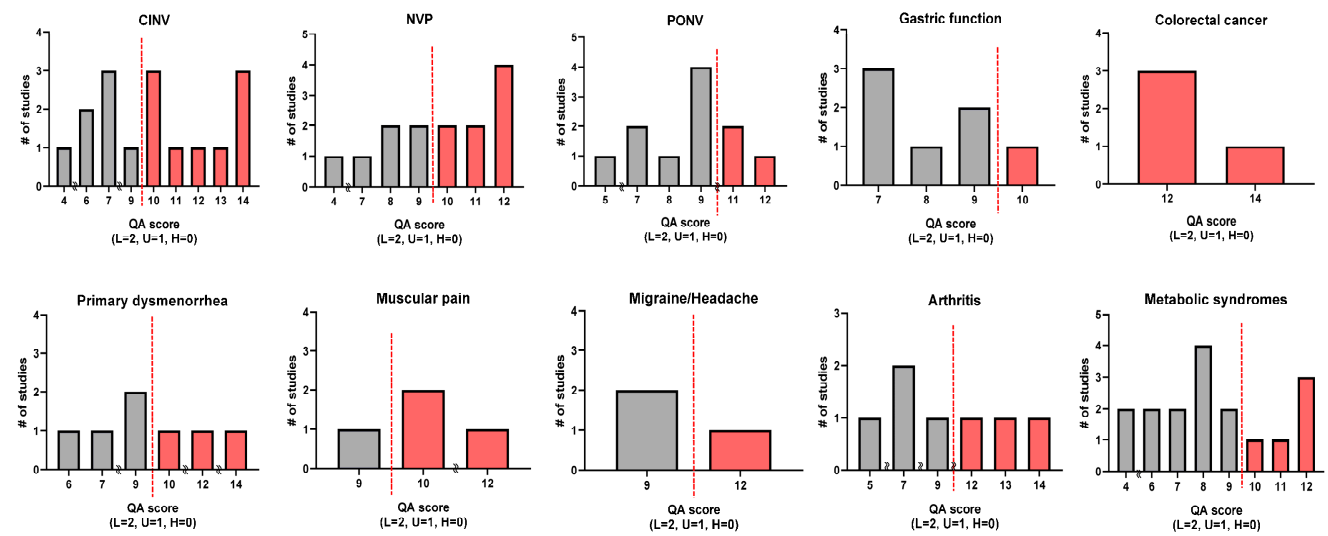

Figure 4. Distribution of ginger randomized controlled trials (RCTs) based on quality assessment (QA) score: a bright color indicates a 'high quality of evidence,' whereas a dark color indicates the opposite. (a) In total, 43 RCTs were addressed to have a high quality of evidence, and (b) each important function was evaluated (CINV: chemotherapy-induced nausea and vomiting; NVP: nausea and vomiting of pregnancy; PONV: postoperative nausea and vomiting). 


\section{Discussion}

The clinical applications of ginger as a medicinal or adjuvant therapy have been receiving significant attention due to its several expected functions, general use globally, and empirically guaranteed safety $[69,70]$. However, an overall comparison of the studies dealing with different functional effects and studies examining minor functions has yet to be adequately performed. As demonstrated by our assessment on reporting quality, attrition bias, and reporting bias were observed to be minor in a majority of studies, and the blinding of participants was also relatively well managed. However, several studies did not describe the method for random sequence generation, method of concealment in allocating procedure, and the blinding of assessors in detail. As a result, several ginger clinical studies had a substantial likelihood of selection and detection bias. For example, in a study by Vladimir et al., the ginger-treated group and the diclofenac-treated group received different formulations of drugs, which might have provided information about allocation with high probability [71]. Finally, only eight studies were judged as having low risks for every type of bias based on the Cochrane Collaboration's quality assessment tool $[14,18,20,28,29,31,45,55]$. Regarding the reporting quality of essential functions, 43 RCTs out of 109 (39.4\%) reported a high quality of evidence. Collectively, no major function satisfied the criteria for every study except colorectal cancer. Major functions that indicated a high quality of evidence in more than half of their studies were CINV, NVP, colorectal cancer, and muscular pain. Less than one-third of PONV, gastric function and metabolic syndrome investigations were appraised to have a high quality of evidence.

Only 17 the included RCTs (15.6\%) presented adverse response information. There were no life-threatening or severe cases reported. Heartburn was the only symptom consistently reported in 16 studies where the participants received between 500 and $2000 \mathrm{mg}$ of ginger per day. This result was supported by the biological property of ginger's constituents inhibiting cyclooxygenase, which has a role in gastric mucosal defense [9,72]. Other reported symptoms could not be generalized as side effects of ginger because the number of reporting studies and the number of participants in each study were both small. For example, a study by Kashefi et al. reported diarrhea as an adverse event in the ginger treatment group. However, only one out of 46 patients $(2.17 \%)$ reported the symptom, indicating no statistically significant correlation to ginger [28]. Moreover, the ratio of reported unexpected cases per group varied considerably between studies. A methodological description regarding the evaluation system used for adverse effects should be provided in future studies for accurate data integration.

Ginger had been investigated as an additional or alternative treatment to standard regimens in fifteen clinical trials of CINV, but the results between studies were controversial, with only about half of them demonstrating significant effects corresponding to the results of previous systematic reviews [73,74]. Moreover, studies from Yunes et al. and Müzeyyen et al., where they concluded the antiemetic effect of ginger, were assessed to have a high risk of bias in the blinding of participants $[75,76]$. The dosage of ginger varied among the studies without any correlation with outcomes. Hence, appropriately designed ginger clinical studies on CINV need to be performed for apparent demonstration. On the contrary, ginger showed a promising effect on NVP. All 10 RCTs on NVP concluded that ginger is as effective as other antiemetic medications such as pyridoxine, metoclopramide or dimenhydrinate or more effective than the placebo at least in some aspects with a dosage less than $1.5 \mathrm{~g}$. An observation study from Portnoi et al. reported that a group of pregnant women showed no statistical differences in teratogenicity when compared to a group receiving nonteratogenic drugs [77]. A large population-based cohort from the Norwegian Mother and Child Cohort study correspondingly reported that ginger did not affect the risk of teratogenicity and abnormalities in birth [78]. Moreover, a review from Stanisiere et al. found that there were no severe side effects with ginger consumption in controlled, uncontrolled, and pre-clinical studies, with a significant reduction in nausea and vomiting [79]. These results support the idea that ginger may be an alternative approach for antiemetic therapy in women during the gestation period of pregnancy. The effectiveness of ginger on PONV, similar to the results from CINV, was inconsistent 
between studies even with a comparatively high dose $(2 \mathrm{~g})$, indicating that further investigations are imperative $[60,80,81]$.

Studies on digestive function mostly reported a positive effect of ginger in reducing gastric emptying time and dysrhythmia. However, symptoms and evaluation methods varied among studies, with substandard general qualities, making it challenging to integrate outcomes. Investigations on the anticancer activity of ginger or its active constituents, especially shogaols and gingerol species, have been conducted in various types of cancer with different models $[6,82]$. However, there was no direct evaluation of the incidence or survival of patients in the four trials that evaluated ginger's effect on colorectal cancer. Instead, factors of inflammation, proliferation, differentiation, and apoptosis were measured upon treatment with 1-2 g of ginger to evaluate the improvement of risk. All four studies reported that colorectal cancer-related risk factors were decreased in the ginger treatment group. Even though only four studies were assessed for this review in regard to colorectal cancer, the results were promising, and the quality of the studies was high, with a low possibility of any bias. Accordingly, further clinical trials are imperative to reinforce the colorectal cancer-preventive effect of ginger.

The analgesic effect of ginger was primarily examined on the alleviation of primary dysmenorrhea. Six studies reported that ginger improved pain relief and had similar effectiveness with medications such as mefenamic acid and ibuprofen compared to the placebo group, except for a study from Marjan et al., where ginger was reported to have no benefit [65]. However, the comparison group in this study managed dysmenorrhea with exercise instead of a placebo or other medication, thus giving a high risk of confounding factors. Furthermore, the dosage was $250 \mathrm{mg}$ a day, whereas the treatment dose ranged between 750 and $1500 \mathrm{mg}$ a day in the other six studies. Altogether, the analgesic effect of ginger on primary dysmenorrhea is worthy of consideration, but more appropriately designed studies should be conducted because the overall quality of the studies was not high. Other types of pain were also improved with ginger treatment in most of the studies. This may be an indicative result from the previously known mechanisms of ginger's active compounds to suppress cyclooxygenase and lipoxygenase [9,72].

The effectiveness of ginger in anti-inflammation and metabolic syndromes has been well studied. For instance, it has been found to significantly reduce symptoms in patients with arthritis-related diseases. Nevertheless, the benefit of ginger among the studies was inconsistent in terms of effectiveness. Noticeably, several studies compared the effectiveness of ginger with other anti-inflammatory drugs. One study concluded that ginger is as effective as ibuprofen in reducing the symptoms of OA, while another study reported the opposite result $[83,84]$. However, all the included studies were conducted with a sample size of less than 100 participants in each group. In addition, the dosages among the studies varied from 15 to $750 \mathrm{mg}$, and the treatment duration ranged from three weeks to 12 weeks. Therefore, further studies with larger sample sizes and standardized study designs should be conducted to confirm the effect of ginger on the symptoms of OA. Regarding metabolic diseases, many studies have demonstrated that ginger can improve blood biochemical parameters and lipid profiles, which can additionally help in reducing the risk of cardiovascular diseases. For instance, ginger supplementation has been found to notably reduce fasting blood sugar, $\mathrm{HbA} 1 \mathrm{c}$, and insulin resistance [85-87]. Additionally, lipid profiles (e.g., total cholesterol, and LDL-C), C-reactive protein, obesity-related cardiovascular risk factors have been found to reduce with ginger intake [24,88-90]. In general, ginger has been found to indicate a beneficial effect on high dosage and long-term treatment in metabolic diseases. However, an obvious limitation is that all studies were performed in a sample size of fewer than 50 participants. Further investigations should be conducted to validate the effect of ginger on metabolic syndrome.

Additionally, the shortcomings in the current ginger clinical trials across domains need to be specified. First, the group size was generally small and rarely exceeded 100 participants per group (only five studies) $[30,37,80,91,92]$. Thirty-six studies conducted clinical trials group sizes of less than 20 patients. Therefore, the drawn conclusions from these studies possess the risk of being underpowered. 
Secondly, the evaluation systems of some symptoms varied between studies that focused on a similar subject. For example, the beneficial effect on digestive and colorectal cancer-preventive function was consistently reported in related studies. However, the different evaluation parameters and markers made it challenging to integrate the outcomes. Finally, we cannot rule out the possibility of low external validity due to the diversity in ethnicity: There were 46 trials from Iran and 18 trials from the United States.

\section{Conclusions}

Ginger is a natural spice that is used in diverse regions to add a pungent flavor to food. Furthermore, ginger has been used as an herbal medicine for common health problems. This systematic review is the first study that has exclusively collected RCTs regarding the efficiency of ginger in several human health conditions. The clinical effects of ginger have been introduced as six subsections: nausea and vomiting, gastrointestinal function, pain, inflammation, metabolic syndromes, and other symptoms. Reportedly, ginger has been effective in a majority of studies, including those that examined the alleviation of NVP, digestive function, improvement in the expression level of markers for colorectal cancer risk, and anti-inflammatory functions. Several other functions have also been regarded as beneficial in trials, with some confronting results. However, a few drawbacks regarding the quality of the trials, inconsistent evaluation systems or parameters, and the generally small size of the studies need to be noted. Therefore, systematically designed research with detailed descriptions of methodology and a sufficient pool of participants is necessary for future clinical trials to address the functional characteristics of ginger.

Supplementary Materials: The following are available online at http://www.mdpi.com/2072-6643/12/1/157/s1, Table S1: PRISMA checklist, Table S2: Study design and demographic description of the included studies with a quality score less than 10, Table S3: Evaluation system and key finding of the included studies with a quality score less than 10, Table S4: Types of adverse effect, incidence rate, and dosage from 17 trials, Table S5: Quality assessment outcomes and quality assessment scores of the included studies.

Author Contributions: S.W.K. supervised the project. S.W.K., N.H.A., S.J.K, and N.P.L. participated in the design of the study. N.H.A., S.J.K., and N.P.L. searched and collected the data. N.H.A., S.J.K., N.P.L., J.E.M., Y.C.Y., E.G.L., M.K., T.J.K., Y.Y.Y., E.Y.S., S.J.Y., N.C.D., and H.M.K. conducted data quality checks, data synthesis, and data interpretation. N.H.A., S.J.K., and N.P.L. performed data processing and statistical analysis. N.H.A., S.J.K., and N.P.L. contributed to drafting the first version of the manuscript. All authors have read, revised critically, and approved the final manuscript. N.H.A. and S.J.K. contributed equally to this work. All authors have read and agreed to the published version of the manuscript.

Funding: This research was supported by the Rural Development Administration of Korea (PJ01420102).

Acknowledgments: This work was supported by BK21 Plus Program in 2019. Graphic abstract was created with Biorender.com.

Conflicts of Interest: The authors declare no conflict of interest.

\section{References}

1. Li, H.; Liu, Y.; Luo, D.; Ma, Y.; Zhang, J.; Li, M.; Yao, L.; Shi, X.; Liu, X.; Yang, K. Ginger for health care: An overview of systematic reviews. Complement. Ther. Med. 2019, 45, 114-123. [CrossRef] [PubMed]

2. Weimer, K.; Schulte, J.; Maichle, A.; Muth, E.R.; Scisco, J.L.; Horing, B.; Enck, P.; Klosterhalfen, S. Effects of ginger and expectations on symptoms of nausea in a balanced placebo design. PLoS ONE 2012, 7, e49031. [CrossRef] [PubMed]

3. Ensiyeh, J.; Sakineh, M.-A.C. Comparing ginger and vitamin B6 for the treatment of nausea and vomiting in pregnancy: A randomised controlled trial. Midwifery 2009, 25, 649-653. [CrossRef] [PubMed]

4. Sharifzadeh, F.; Kashanian, M.; Koohpayehzadeh, J.; Rezaian, F.; Sheikhansari, N.; Eshraghi, N. A comparison between the effects of ginger, pyridoxine (vitamin B6) and placebo for the treatment of the first trimester nausea and vomiting of pregnancy (NVP). J. Matern. Fetal Neonatal Med. 2018, 31, 2509-2514. [CrossRef] [PubMed] 
5. Ali, B.H.; Blunden, G.; Tanira, M.O.; Nemmar, A. Some phytochemical, pharmacological and toxicological properties of ginger (Zingiber officinale Roscoe): A review of recent research. Food Chem. Toxicol. 2008, 46, 409-420. [CrossRef] [PubMed]

6. Mahomoodally, M.F.; Aumeeruddy, M.Z.; Rengasamy, K.R.R.; Roshan, S.; Hammad, S.; Pandohee, J.; $\mathrm{Hu}, \mathrm{X}$.; Zengin, G. Ginger and its active compounds in cancer therapy: From folk uses to nano-therapeutic applications. Semin. Cancer Biol. 2019. [CrossRef]

7. Kubra, I.R.; Rao, L.J.M. An impression on current developments in the technology, chemistry, and biological activities of ginger (Zingiber officinale Roscoe). Crit. Rev. Food Sci. Nutr. 2012, 52, 651-688. [CrossRef]

8. Nile, S.H.; Park, S.W. Chromatographic analysis, antioxidant, anti-inflammatory, and xanthine oxidase inhibitory activities of ginger extracts and its reference compounds. Ind. Crop. Prod. 2015, 70, 238-244. [CrossRef]

9. Mao, Q.-Q.; Xu, X.-Y.; Cao, S.-Y.; Gan, R.-Y.; Corke, H.; Beta, T.; Li, H.-B. Bioactive compounds and bioactivities of ginger (Zingiber officinale Roscoe). Foods 2019, 8, 185. [CrossRef]

10. Crichton, M.; Marshall, S.; Marx, W.; McCarthy, A.L.; Isenring, E. Efficacy of ginger (Zingiber officinale) in ameliorating chemotherapy-induced nausea and vomiting and chemotherapy-related outcomes: A systematic review update and meta-analysis. J. Acad. Nutr. Diet. 2019. [CrossRef]

11. Chen, C.X.; Barrett, B.; Kwekkeboom, K.L. Efficacy of oral ginger (Zingiber officinale) for dysmenorrhea: A systematic review and meta-analysis. Evid.-Based Complement. Altern. Med. 2016, 2016, 10. [CrossRef] [PubMed]

12. Zhu, J.; Chen, H.; Song, Z.; Wang, X.; Sun, Z. Effects of ginger (Zingiber officinale Roscoe) on type 2 diabetes mellitus and components of the metabolic syndrome: A systematic review and meta-analysis of randomized controlled trials. Evid.-Based Complement. Altern. Med. 2018, 2018, 5692962. [CrossRef] [PubMed]

13. Mozaffari-Khosravi, H.; Naderi, Z.; Dehghan, A.; Nadjarzadeh, A.; Fallah Huseini, H. Effect of ginger supplementation on proinflammatory cytokines in older patients with osteoarthritis: Outcomes of a randomized controlled clinical trial. J. Nutr. Gerontol. Geriatr. 2016, 35, 209-218. [CrossRef] [PubMed]

14. Jiang, Y.; Turgeon, D.K.; Wright, B.D.; Sidahmed, E.; Ruffin, M.T.; Brenner, D.E.; Sen, A.; Zick, S.M. Effect of ginger root on cyclooxygenase-1 and 15-hydroxyprostaglandin dehydrogenase expression in colonic mucosa of humans at normal and increased risk for colorectal cancer. Eur. J. Cancer Prev. 2013, 22, 455-460. [CrossRef] [PubMed]

15. Moher, D.; Liberati, A.; Tetzlaff, J.; Altman, D.G. Preferred reporting items for systematic reviews and meta-analyses: The PRISMA statement. BMJ 2009, 339, b2535. [CrossRef]

16. Higgins, J.P.T.; Altman, D.G.; Gøtzsche, P.C.; Jüni, P.; Moher, D.; Oxman, A.D.; Savović, J.; Schulz, K.F.; Weeks, L.; Sterne, J.A.C. The cochrane collaboration's tool for assessing risk of bias in randomised trials. BMJ 2011, 343, d5928. [CrossRef]

17. Marx, W.; McCarthy, A.L.; Ried, K.; McKavanagh, D.; Vitetta, L.; Sali, A.; Lohning, A.; Isenring, E. The effect of a standardized ginger extract on chemotherapy-induced nausea-related quality of life in patients undergoing moderately or highly emetogenic chemotherapy: A double blind, randomized, placebo controlled trial. Nutrients 2017, 9, 867. [CrossRef]

18. Sanaati, F.; Najafi, S.; Kashaninia, Z.; Sadeghi, M. Effect of ginger and chamomile on nausea and vomiting caused by chemotherapy in Iranian women with breast cancer. Asian Pac. J. Cancer Prev. 2016, 17, 4125-4129.

19. Thamlikitkul, L.; Srimuninnimit, V.; Akewanlop, C.; Ithimakin, S.; Techawathanawanna, S.; Korphaisarn, K.; Chantharasamee, J.; Danchaivijitr, P.; Soparattanapaisarn, N. Efficacy of ginger for prophylaxis of chemotherapy-induced nausea and vomiting in breast cancer patients receiving adriamycincyclophosphamide regimen: A randomized, double-blind, placebo-controlled, crossover study. Support Care Cancer 2017, 25, 459-464. [CrossRef]

20. Li, X.; Qin, Y.; Liu, W.; Zhou, X.Y.; Li, Y.N.; Wang, L.Y. Efficacy of ginger in ameliorating acute and delayed chemotherapy-induced nausea and vomiting among patients with lung cancer receiving cisplatin-based regimens: A randomized controlled trial. Integr. Cancer 2018, 17, 747-754. [CrossRef]

21. Ansari, M.; Porouhan, P.; Mohammadianpanah, M.; Omidvari, S.; Mosalaei, A.; Ahmadloo, N.; Nasrollahi, H.; Hamedi, S.H. Efficacy of ginger in control of chemotherapy induced nausea and vomiting in breast cancer patients receiving doxorubicin-based chemotherapy. Asian Pac. J. Cancer Prev. 2016, 17, 3877-3880. [PubMed]

22. Matsumura, M.D.; Zavorsky, G.S.; Smoliga, J.M. The effects of pre-exercise ginger supplementation on muscle damage and delayed onset muscle soreness. Phytother. Res. 2015, 29, 887-893. [CrossRef] [PubMed] 
23. Martins, L.B.; Rodrigues, A.; Rodrigues, D.F.; Dos Santos, L.C.; Teixeira, A.L.; Ferreira, A.V.M. Double-blind placebo-controlled randomized clinical trial of ginger (Zingiber officinale Rosc) addition in migraine acute treatment. Cephalalgia 2019, 39, 68-76. [CrossRef] [PubMed]

24. Makhdoomi Arzati, M.; Mohammadzadeh Honarvar, N.; Saedisomeolia, A.; Anvari, S.; Effatpanah, M.; Makhdoomi Arzati, R.; Yekaninejad, M.S.; Hashemi, R.; Djalali, M. The effects of ginger on fasting blood sugar, hemoglobin A1c, and lipid profiles in patients with type 2 diabetes. Int. J. Endocrinol. Metab. 2017, 15, e57927. [CrossRef]

25. Ebrahimzadeh Attari, V.; Ostadrahimi, A.; Asghari Jafarabadi, M.; Mehralizadeh, S.; Mahluji, S. Changes of serum adipocytokines and body weight following zingiber officinale supplementation in obese women: A RCT. Eur. J. Nutr. 2016, 55, 2129-2136. [CrossRef]

26. Ebrahimzadeh Attari, V.; Asghari Jafarabadi, M.; Zemestani, M.; Ostadrahimi, A. Effect of zingiber officinale supplementation on obesity management with respect to the uncoupling protein $1-3826 \mathrm{~A}>\mathrm{G}$ and B3-adrenergic receptor Trp64Arg polymorphism. Phytother. Res. 2015, 29, 1032-1039. [CrossRef]

27. Aryaeian, N.; Shahram, F.; Mahmoudi, M.; Tavakoli, H.; Yousefi, B.; Arablou, T.; Jafari Karegar, S. The effect of ginger supplementation on some immunity and inflammation intermediate genes expression in patients with active rheumatoid arthritis. Gene 2019, 698, 179-185. [CrossRef]

28. Kashefi, F.; Khajehei, M.; Alavinia, M.; Golmakani, E.; Asili, J. Effect of ginger (Zingiber officinale) on heavy menstrual bleeding: A placebo-controlled, randomized clinical trial. Phytother. Res. 2015, 29, 114-119. [CrossRef]

29. Paritakul, P.; Ruangrongmorakot, K.; Laosooksathit, W.; Suksamarnwong, M.; Puapornpong, P. The effect of ginger on breast milk volume in the early postpartum period: A randomized, double-blind controlled trial. Breastfeed. Med. 2016, 11, 361-365. [CrossRef]

30. Ryan, J.L.; Heckler, C.E.; Roscoe, J.A.; Dakhil, S.R.; Kirshner, J.; Flynn, P.J.; Hickok, J.T.; Morrow, G.R. Ginger (Zingiber officinale) reduces acute chemotherapy-induced nausea: A URCC CCOP study of 576 patients. Support Care Cancer 2012, 20, 1479-1489. [CrossRef]

31. Zick, S.M.; Ruffin, M.T.; Lee, J.; Normolle, D.P.; Siden, R.; Alrawi, S.; Brenner, D.E. Phase II trial of encapsulated ginger as a treatment for chemotherapy-induced nausea and vomiting. Support Care Cancer 2009, 17, 563-572. [CrossRef] [PubMed]

32. Fahimi, F.; Khodadad, K.; Amini, S.; Naghibi, F.; Salamzadeh, J.; Baniasadi, S. Evaluating the effect of zingiber officinalis on nausea and vomiting in patients receiving cisplatin based regimens. Iran. J. Pharm. Res. 2011, 10, 379-384. [PubMed]

33. Yekta, Z.P.; Ebrahimi, S.M.; Hosseini, M.; Nasrabadi, A.N.; Sedighi, S.; Surmaghi, M.H.; Madani, H. Ginger as a miracle against chemotherapy-induced vomiting. Iran. J. Nurs. Midwifery Res. 2012, 17, 325-329. [PubMed]

34. Willetts, K.E.; Ekangaki, A.; Eden, J.A. Effect of a ginger extract on pregnancy-induced nausea: A randomised controlled trial. Aust. N. Z. J. Obstet. Gynaecol. 2003, 43, 139-144. [CrossRef] [PubMed]

35. Vutyavanich, T.; Kraisarin, T.; Ruangsri, R. Ginger for nausea and vomiting in pregnancy: Randomized, double-masked, placebo-controlled trial. Obstet. Gynecol. 2001, 97, 577-582. [CrossRef]

36. Fischer-Rasmussen, W.; Kjaer, S.K.; Dahl, C.; Asping, U. Ginger treatment of hyperemesis gravidarum. Eur. J. Obstet. Gynecol. Reprod. Biol. 1991, 38, 19-24. [CrossRef]

37. Smith, C.; Crowther, C.; Willson, K.; Hotham, N.; McMillian, V. A randomized controlled trial of ginger to treat nausea and vomiting in pregnancy. Obstet. Gynecol. 2004, 103, 639-645. [CrossRef]

38. Biswas, S.C.; Dey, R.; Kamliya, G.S.; Bal, R.; Hazra, A.; Tripathi, S. A single-masked, randomized, controlled trial of ginger extract in the treatment of nausea and vomiting of pregnancy. J. Int. Med. Sci. Acad. 2011, 24, 167-169.

39. Firouzbakht, M.; Nikpour, M.; Jamali, B.; Omidvar, S. Comparison of ginger with vitamin B6 in relieving nausea and vomiting during pregnancy. Ayu 2014, 35, 289-293. [CrossRef]

40. Arfeen, Z.; Owen, H.; Plummer, J.L.; Ilsley, A.H.; Sorby-Adams, R.A.; Doecke, C.J. A double-blind randomized controlled trial of ginger for the prevention of postoperative nausea and vomiting. Anaesth. Intensive Care 1995, 23, 449-452. [CrossRef]

41. Eberhart, L.H.; Mayer, R.; Betz, O.; Tsolakidis, S.; Hilpert, W.; Morin, A.M.; Geldner, G.; Wulf, H.; Seeling, W. Ginger does not prevent postoperative nausea and vomiting after laparoscopic surgery. Anesth. Analg. 2003, 96, 995-998. [CrossRef] [PubMed] 
42. Mandal, P.; Das, A.; Majumdar, S.; Bhattacharyya, T.; Mitra, T.; Kundu, R. The efficacy of ginger added to ondansetron for preventing postoperative nausea and vomiting in ambulatory surgery. Pharmacogn. Res 2014, 6, 52-57. [CrossRef]

43. Ozgoli, G.; Goli, M.; Moattar, F. Comparison of effects of ginger, mefenamic acid, and ibuprofen on pain in women with primary dysmenorrhea. J. Altern. Complement. Med. 2009, 15, 129-132. [CrossRef] [PubMed]

44. Kashefi, F.; Khajehei, M.; Tabatabaeichehr, M.; Alavinia, M.; Asili, J. Comparison of the effect of ginger and zinc sulfate on primary dysmenorrhea: A placebo-controlled randomized trial. Pain Manag. Nurs. 2014, 15, 826-833. [CrossRef] [PubMed]

45. Rahnama, P.; Montazeri, A.; Huseini, H.F.; Kianbakht, S.; Naseri, M. Effect of zingiber officinale R. rhizomes (ginger) on pain relief in primary dysmenorrhea: A placebo randomized trial. BMC Complement. Altern. Med. 2012, 12, 92. [CrossRef] [PubMed]

46. Black, C.D.; O'Connor, P.J. Acute effects of dietary ginger on muscle pain induced by eccentric exercise. Phytother. Res. 2010, 24, 1620-1626. [CrossRef]

47. Black, C.D.; Herring, M.P.; Hurley, D.J.; O'Connor, P.J. Ginger (Zingiber officinale) reduces muscle pain caused by eccentric exercise. J. Pain 2010, 11, 894-903. [CrossRef]

48. Mahluji, S.; Attari, V.E.; Mobasseri, M.; Payahoo, L.; Ostadrahimi, A.; Golzari, S.E. Effects of ginger (Zingiber officinale) on plasma glucose level, HbA1c and insulin sensitivity in type 2 diabetic patients. Int. J. Food Sci. Nutr. 2013, 64, 682-686. [CrossRef]

49. Khandouzi, N.; Shidfar, F.; Rajab, A.; Rahideh, T.; Hosseini, P.; Mir Taheri, M. The effects of ginger on fasting blood sugar, hemoglobin a1c, apolipoprotein B, apolipoprotein a-I and malondialdehyde in type 2 diabetic patients. Iran. J. Pharm. Res. 2015, 14, 131-140.

50. Phillips, S.; Hutchinson, S.; Ruggier, R. Zingiber officinale does not affect gastric emptying rate. A randomised, placebo-controlled, crossover trial. Anaesthesia 1993, 48, 393-395. [CrossRef]

51. Citronberg, J.; Bostick, R.; Ahearn, T.; Turgeon, D.K.; Ruffin, M.T.; Djuric, Z.; Sen, A.; Brenner, D.E.; Zick, S.M. Effects of ginger supplementation on cell-cycle biomarkers in the normal-appearing colonic mucosa of patients at increased risk for colorectal cancer: Results from a pilot, randomized, and controlled trial. Cancer Prev. Res. 2013, 6, 271-281. [CrossRef] [PubMed]

52. Zick, S.M.; Turgeon, D.K.; Vareed, S.K.; Ruffin, M.T.; Litzinger, A.J.; Wright, B.D.; Alrawi, S.; Normolle, D.P.; Djuric, Z.; Brenner, D.E. Phase II study of the effects of ginger root extract on eicosanoids in colon mucosa in people at normal risk for colorectal cancer. Cancer Prev. Res. 2011, 4, 1929-1937. [CrossRef] [PubMed]

53. Zick, S.M.; Turgeon, D.K.; Ren, J.; Ruffin, M.T.; Wright, B.D.; Sen, A.; Djuric, Z.; Brenner, D.E. Pilot clinical study of the effects of ginger root extract on eicosanoids in colonic mucosa of subjects at increased risk for colorectal cancer. Mol. Carcinog. 2015, 54, 908-915. [CrossRef]

54. Van Tilburg, M.A.L.; Palsson, O.S.; Ringel, Y.; Whitehead, W.E. Is ginger effective for the treatment of irritable bowel syndrome? A double blind randomized controlled pilot trial. Complement. Ther. Med. 2014, 22, 17-20. [CrossRef] [PubMed]

55. Wigler, I.; Grotto, I.; Caspi, D.; Yaron, M. The effects of zintona EC (a ginger extract) on symptomatic gonarthritis. Osteoarthr. Cartil. 2003, 11, 783-789. [CrossRef]

56. Pillai, A.K.; Sharma, K.K.; Gupta, Y.K.; Bakhshi, S. Anti-emetic effect of ginger powder versus placebo as an add-on therapy in children and young adults receiving high emetogenic chemotherapy. Pediatr. Blood Cancer 2011, 56, 234-238. [CrossRef]

57. Konmun, J.; Danwilai, K.; Ngamphaiboon, N.; Sripanidkulchai, B.; Sookprasert, A.; Subongkot, S. A phase II randomized double-blind placebo-controlled study of 6-gingerol as an anti-emetic in solid tumor patients receiving moderately to highly emetogenic chemotherapy. Med. Oncol. 2017, 34, 69. [CrossRef]

58. Apariman, S.; Ratchanon, S.; Wiriyasirivej, B. Effectiveness of ginger for prevention of nausea and vomiting after gynecological laparoscopy. J. Med. Assoc. Thai. 2006, 89, 2003-2009.

59. Chaiyakunapruk, N.; Kitikannakorn, N.; Nathisuwan, S.; Leeprakobboon, K.; Leelasettagool, C. The efficacy of ginger for the prevention of postoperative nausea and vomiting: A meta-analysis. Am. J. Obstet. Gynecol. 2006, 194, 95-99. [CrossRef]

60. Phillips, S.; Ruggier, R.; Hutchinson, S.E. Zingiber officinale (ginger)—An antiemetic for day case surgery. Anaesthesia 1993, 48, 715-717. [CrossRef] 
61. Bone, M.E.; Wilkinson, D.J.; Young, J.R.; McNeil, J.; Charlton, S. Ginger root-A new antiemetic. The effect of ginger root on postoperative nausea and vomiting after major gynaecological surgery. Anaesthesia 1990, 45, 669-671. [CrossRef] [PubMed]

62. Dabaghzadeh, F.; Khalili, H.; Dashti-Khavidaki, S.; Abbasian, L.; Moeinifard, A. Ginger for prevention of antiretroviral-induced nausea and vomiting: A randomized clinical trial. Expert Opin. Drug Saf. 2014, 13, 859-866. [CrossRef]

63. Lien, H.C.; Sun, W.M.; Chen, Y.H.; Kim, H.; Hasler, W.; Owyang, C. Effects of ginger on motion sickness and gastric slow-wave dysrhythmias induced by circular vection. Am. J. Physiol. Gastrointest. Liver Physiol. 2003, 284, G481-G489. [CrossRef] [PubMed]

64. Gonlachanvit, S.; Chen, Y.H.; Hasler, W.L.; Sun, W.M.; Owyang, C. Ginger reduces hyperglycemia-evoked gastric dysrhythmias in healthy humans: Possible role of endogenous prostaglandins. J. Pharm. Exp. 2003, 307, 1098-1103. [CrossRef] [PubMed]

65. Shirvani, M.A.; Motahari-Tabari, N.; Alipour, A. Use of ginger versus stretching exercises for the treatment of primary dysmenorrhea: A randomized controlled trial. J. Integr. Med. 2017, 15, 295-301. [CrossRef]

66. Maghbooli, M.; Golipour, F.; Moghimi Esfandabadi, A.; Yousefi, M. Comparison between the efficacy of ginger and sumatriptan in the ablative treatment of the common migraine. Phytother. Res. 2014, 28, 412-415. [CrossRef]

67. Kulkarni, R.A.; Deshpande, A.R. Anti-inflammatory and antioxidant effect of ginger in tuberculosis. J. Complement. Integr. Med. 2016, 13, 201-206. [CrossRef]

68. Bordia, A.; Verma, S.K.; Srivastava, K.C. Effect of ginger (Zingiber officinale Rosc.) and fenugreek (Trigonella foenumgraecum L.) on blood lipids, blood sugar and platelet aggregation in patients with coronary artery disease. Prostaglandins Leukot. Essent. Fat. Acids 1997, 56, 379-384. [CrossRef]

69. Marx, W.; Ried, K.; McCarthy, A.L.; Vitetta, L.; Sali, A.; McKavanagh, D.; Isenring, L. Ginger-Mechanism of action in chemotherapy-induced nausea and vomiting: A review. Crit. Rev. Food Sci. Nutr. 2017, 57, 141-146. [CrossRef]

70. Danwilai, K.; Konmun, J.; Sripanidkulchai, B.-O.; Subongkot, S. Antioxidant activity of ginger extract as a daily supplement in cancer patients receiving adjuvant chemotherapy: A pilot study. Cancer Manag. Res. 2017, 9, 11-18. [CrossRef]

71. Drozdov, V.N.; Kim, V.A.; Tkachenko, E.V.; Varvanina, G.G. Influence of a specific ginger combination on gastropathy conditions in patients with osteoarthritis of the knee or hip. J. Altern. Complement. Med. 2012, 18, 583-588. [CrossRef]

72. Grzanna, R.; Lindmark, L.; Frondoza, C.G. Ginger-An herbal medicinal product with broad antiinflammatory actions. J. Med. Food 2005, 8, 125-132. [CrossRef]

73. Saneei Totmaj, A.; Emamat, H.; Jarrahi, F.; Zarrati, M. The effect of ginger (Zingiber officinale) on chemotherapy-induced nausea and vomiting in breast cancer patients: A systematic literature review of randomized controlled trials. Phytother. Res. 2019, 33, 1957-1965. [CrossRef] [PubMed]

74. Marx, W.M.; Teleni, L.; McCarthy, A.L.; Vitetta, L.; McKavanagh, D.; Thomson, D.; Isenring, E. Ginger (Zingiber officinale) and chemotherapy-induced nausea and vomiting: A systematic literature review. Nutr. Rev. 2013, 71, 245-254. [CrossRef] [PubMed]

75. Panahi, Y.; Saadat, A.; Sahebkar, A.; Hashemian, F.; Taghikhani, M.; Abolhasani, E. Effect of ginger on acute and delayed chemotherapy-induced nausea and vomiting: A pilot, randomized, open-label clinical trial. Integr. Cancer 2012, 11, 204-211. [CrossRef] [PubMed]

76. Arslan, M.; Ozdemir, L. Oral intake of ginger for chemotherapy-induced nausea and vomiting among women with breast cancer. Clin. J. Oncol. Nurs. 2015, 19, E92-E97. [CrossRef] [PubMed]

77. Portnoi, G.; Chng, L.-A.; Karimi-Tabesh, L.; Koren, G.; Tan, M.P.; Einarson, A. Prospective comparative study of the safety and effectiveness of ginger for the treatment of nausea and vomiting in pregnancy. Am. J. Obstet. Gynecol. 2003, 189, 1374-1377. [CrossRef]

78. Heitmann, K.; Nordeng, H.; Holst, L. Safety of ginger use in pregnancy: Results from a large population-based cohort study. Eur. J. Clin. Pharmacol. 2013, 69, 269-277. [CrossRef]

79. Stanisiere, J.; Mousset, P.-Y.; Lafay, S. How safe is ginger rhizome for decreasing nausea and vomiting in women during early pregnancy? Foods 2018, 7, 50. [CrossRef] 
80. Kalava, A.; Darji, S.J.; Kalstein, A.; Yarmush, J.M.; SchianodiCola, J.; Weinberg, J. Efficacy of ginger on intraoperative and postoperative nausea and vomiting in elective cesarean section patients. Eur. J. Obstet. Gynecol. Reprod. Biol. 2013, 169, 184-188. [CrossRef]

81. Gillart, T.; Bazin, J.E.; Montetagaud, M.; Bevillard, F.; Amara, S.; Schoeffler, P. The effects of volume and speed of injection in peribulbar anaesthesia. Anaesthesia 1998, 53, 486-491. [CrossRef]

82. Prasad, S.; Tyagi, A.K. Ginger and its constituents: Role in prevention and treatment of gastrointestinal cancer. Gastroenterol. Res. Pract. 2015, 2015, 11. [CrossRef]

83. Paramdeep, G. Efficacy and tolerability of ginger (Zingiber officinale) in patients of osteoarthritis of knee. Indian J. Physiol. Pharm. 2013, 57, 177-183.

84. Bliddal, H.; Rosetzsky, A.; Schlichting, P.; Weidner, M.S.; Andersen, L.A.; Ibfelt, H.H.; Christensen, K.; Jensen, O.N.; Barslev, J. A randomized, placebo-controlled, cross-over study of ginger extracts and ibuprofen in osteoarthritis. Osteoarthr. Cartil. 2000, 8, 9-12. [CrossRef]

85. Shidfar, F.; Rajab, A.; Rahideh, T.; Khandouzi, N.; Hosseini, S.; Shidfar, S. The effect of ginger (Zingiber officinale) on glycemic markers in patients with type 2 diabetes. J. Complement. Integr. Med. 2015, 12, 165-170. [CrossRef] [PubMed]

86. Arablou, T.; Aryaeian, N.; Valizadeh, M.; Sharifi, F.; Hosseini, A.; Djalali, M. The effect of ginger consumption on glycemic status, lipid profile and some inflammatory markers in patients with type 2 diabetes mellitus. Int. J. Food Sci. Nutr. 2014, 65, 515-520. [CrossRef] [PubMed]

87. Mozaffari-Khosravi, H.; Talaei, B.; Jalali, B.-A.; Najarzadeh, A.; Mozayan, M.R. The effect of ginger powder supplementation on insulin resistance and glycemic indices in patients with type 2 diabetes: A randomized, double-blind, placebo-controlled trial. Complement. Ther. Med. 2014, 22, 9-16. [CrossRef] [PubMed]

88. Tabibi, H.; Imani, H.; Atabak, S.; Najafi, I.; Hedayati, M.; Rahmani, L. Effects of ginger on serum lipids and lipoproteins in peritoneal dialysis patients: A randomized controlled trial. Perit. Dial. Int. 2016, 36, 140-145. [CrossRef]

89. Andallu, B.; Radhika, B.; Suryakantham, V. Effect of aswagandha, ginger and mulberry on hyperglycemia and hyperlipidemia. Plant Foods Hum. Nutr. 2003, 58, 1-7. [CrossRef]

90. Karimi, N.; Dabidi Roshan, V.; Fathi Bayatiyani, Z. Individually and combined water-based exercise with ginger supplement, on systemic inflammation and metabolic syndrome indices, among the obese women with breast neoplasms. Iran. J. Cancer Prev. 2015, 8, e3856. [CrossRef]

91. Adib Rad, H.; Basirat, Z.; Bakouei, F.; Moghadamnia, A.A.; Khafri, S.; Farhadi Kotenaei, Z.; Nikpour, M.; Kazemi, S. Effect of ginger and novafen on menstrual pain: A cross-over trial. Taiwan J. Obstet. Gynecol. 2018, 57, 806-809. [CrossRef] [PubMed]

92. Bossi, P.; Cortinovis, D.; Fatigoni, S.; Cossu Rocca, M.; Fabi, A.; Seminara, P.; Ripamonti, C.; Alfieri, S.; Granata, R.; Bergamini, C.; et al. A randomized, double-blind, placebo-controlled, multicenter study of a ginger extract in the management of chemotherapy-induced nausea and vomiting (CINV) in patients receiving high-dose cisplatin. Ann. Oncol. 2017, 28, 2547-2551. [CrossRef] [PubMed]

(C) 2020 by the authors. Licensee MDPI, Basel, Switzerland. This article is an open access article distributed under the terms and conditions of the Creative Commons Attribution (CC BY) license (http://creativecommons.org/licenses/by/4.0/). 